\title{
One-Step Modal Logics, Intuitionistic and Classical, Part 1
}

\author{
Harold T. Hodes ${ }^{1}$ ii
}

Received: 24 January 2019 / Accepted: 8 September 2020 / Published online: 7 January 2021

(C) Springer Nature B.V. 2021

\begin{abstract}
This paper and its sequel "look under the hood" of the usual sorts of proof-theoretic systems for certain well-known intuitionistic and classical propositional modal logics. Section 1 is preliminary. Of most importance: a marked formula will be the result of prefixing a formula in a propositional modal language with a step-marker, for this paper either $\mathbf{0}$ or $\mathbf{1}$. Think of $\mathbf{1}$ as indicating the taking of "one step away from $\mathbf{0}$." Deductions will be constructed using marked formulas. Section 2 presents the modeltheoretic concepts, based on those in [7], that guide the rest of this paper. Section 3 presents Natural Deduction systems IK and CK, formalizations of intuitionistic and classical one-step versions of K. In these systems, occurrences of step-markers allow deductions to display deductive structure that is covered over in familiar "no step" proof-theoretic systems for such logics. $\square$ and $\diamond$ are governed by Introduction and Elimination rules; the familiar $\mathrm{K}$ rule and Necessitation are derived (i.e. admissible) rules. CK will be the result of adding the $\mathbf{0}$-version of the Rule of Excluded Middle to the rules which generate IK. Note: IK is the result of merely dropping that rule from those generating $\mathrm{CK}$, without addition of further rules or axioms (as was needed in [7]). These proof-theoretic systems yield intuitionistic and classical consequence relations by the obvious definition. Section 4 provides some examples of what can be deduced in IK. Section 5 defines some proof-theoretic concepts that are used in Section 6 to prove the soundness of the consequence relation for IK (relative to the class of models defined in Section 2.) Section 7 proves its completeness (relative to that class). Section 8 extends these results to the consequence relation for CK. (Looking ahead: Part 2 will investigate one-step proof-theoretic systems formalizing intuitionistic and classical one-step versions of some familiar logics stronger than K.)
\end{abstract}

Thanks to Philip Sink, and to the referee, for reading several drafts of this paper and catching many errors.

Harold T. Hodes

hth3@cornell.edu

1 Sage School of Philosophy, Cornell University, Ithaca, NY, USA 
Keywords Intiutionistic and classical modal logics - Introduction and elimination rules · Natural deduction · One-step · Plotikin-Sterling frames and models . Soundness and completeness theorems

Familiar proof-theoretic systems formalizing normal modal logics take as primitive the rule Necessitation, and either the K-axioms (if the systems are Frege-systems) or a K-rule (if they are natural deduction or sequent systems); for the modal logic K, these are the only modal proof-theoretic primitives. This paper will attend to natural deduction systems. The just-mentioned rules may be pictured as follows.

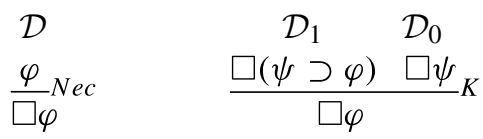

Here $\mathcal{D}_{0}$ and $\mathcal{D}_{1}$ are any deductions in the relevant ND system with the indicated conclusions. But $\mathcal{D}$ must be a deduction with no assumptions; this makes Necessitation a rule of proof rather than a rule of inference.

When contrasted with the primitive rules governing the familiar logical constants, these rules are anomalous in two respects.

(A) A good formalization of a classical logic should transform into a formalization of its natural intuitionistic counterpart merely by removal of a "classicalizing" rule or axiom. But doing this to the usual formalizations of classical $\mathrm{K}$ yield proof-theoretic systems that are too weak; to obtain the natural intuitionistic version of K we need to follow Plotkin and Sterling's lead in [7] by adding either axioms (as in the Frege-system that Plotkin and Sterling actually offered) or rules (if the system is a natural deduction or sequent system) that go beyond Necessitation and the K-rule, and that make use of $\supset, \vee$ and $\perp$, in addition to $\square$ and $\diamond$.

(B) Necessitation differs from familiar introduction rules by virtue of not being a rule of inference. The K-rule doesn't look anything like an elimination rule for $\square$, at least if our paradigm elimination rules are those for \&, つ, $\vee$ and $\perp$.

These two anomalies might make one suspect that the familiar proof-theoretic systems for $\mathrm{K}$ (and for other normal modal logics) hide some important machinery "under the hood". I endorse this suspicion. These anomalies result from formalizing $\mathrm{K}$ (and the other normal modal logics) in a "no step" format ${ }^{1}$, which cuts down on notational clutter at the expense of explicit articulation of deductive structure.

This project is motivated by the thought that the senses of logical constants are constituted by at least some of the deductive rules that govern them. I propose those rules are exactly the introduction and elimination rules (or, in terms of sequent

\footnotetext{
${ }^{1}$ That is, without the step-markers to be introduced below.
} 
calculi of the usual sort, right-entry and left-entry rules). ${ }^{2}$ This is not the place for an extended discussion of these matters. ${ }^{3}$

The status $\square$ and $\diamond$ as logical constants is obscured by proof-theoretic systems in which one cannot "see" their underlying introduction and elimination rules. "One step" proof-theoretic systems, of the sort to be introduced below, give deeper proof-theoretic insight into normal modal logics than is provided by "no step" systems: the primitive rules governing $\square$ and $\diamond$ for both intuitionistic and classical $\mathrm{K}$ will be proper introduction and elimination rules of inference; and classicalization will not render any intuitionistic rules redundant. Similarly for the most popular strengthenings of $\mathrm{K}$.

\section{Preliminaries}

Most mathematical notation and terminology used below will be familar. Natural numbers are finite von-Neumann ordinals. For $n \in \omega$ let $(n)=\{1, \ldots, n\}$.

\subsection{Notation}

Let our logical lexicon $L L=\{\perp, \top, \supset, \&, \vee, \square, \diamond\}$. For what follows we will consider a countable set $S$ of formula (i.e. propositional, sentential) constants. From $L L$ generate the set $F \mathrm{ml}$ of formulas based on $S$ in the usual way.

$\neg$ and $\leftrightarrow$ are defined in the usual way. Note: we could have taken $T$ to be defined, e.g. as $(\perp \supset \perp)$. But $T$ will do work in the primitive rules of the proof-theoretic systems to be discussed below, and this work will not make use of the internal structure

\footnotetext{
${ }^{2}$ For more on this idea, see [3]. This and related ideas have been developed in many places. Regarding $\square$ and $\searrow$, see Andrew Parisi's dissertation [5], which takes a proof-theoretic approach using hypersequents quite different from the approach I take in this paper.

The motivating idea raises the question of what counts as an introduction or an elimination rule. This is not the place for an extended discussion, but let me say this. Determining the canonical "sense-fixing" rules for first-order $\forall$ and $\exists$ is a delicate matter. I think that they should be "free logic" rules, but there are still several ways to go with that. All ways put them in a "local holism" (in the sense of Christopher Peacocke, see [6]) with a primitive logical 1-place predicate for existence. (The more traditional existencepresupposing first-order logics are convenient but philosophically misleading simplifications of their free counterparts.)

In [8] (p. 15), Jason Turner gives an argument that one might think shows that "the" (better: any reasonable choice of) introduction and elimination rules for $\exists$ do not determine its sense. Suffice to say: I think that that impression turns on a misunderstanding of what is required for introduction and elimination rules to be sense-determining.

${ }^{3}$ But I want to digress on one issue. In addition to the introduction and elimination rules governing $\forall$ and $\exists$, their amenability to domain restrictions determined by contexts-of-use may be thought to be built into their senses. One could take this to be a friendly weakening of my motivating thought. And there are various "species" of necessity and possibility which can be expressed by $\square$ and $\diamond$, according to contexts-of-use; and this too may be thought to be built into the senses of $\square$ and $\diamond$. On the other hand, I think it better to construe the domain-restrictablity of $\forall$ and $\exists$ as a matter of logical syntax: the determiners of natural languages that express universal and existential quantification are two-place, of type $((i),(i))$ rather than type $((i))$ (with the first place for a restrictor, which can in principle be null). Is a similar approach to the species of necessity and possibility optimal? This is an interesting question, not to be pursued here; but see [1].
} 
that $\top$ would have under any such definition; taking $\top$ to be defined would make the rules in which $T$ plays a role more complex than they need to be. Furthermore there are infinitely many ways in which $T$ could be defined. Making a choice would make our choice of certain primitive rules arbitrary. ${ }^{4}$ This point does not cut against defining $\neg$ and $\leftrightarrow$, since these will play no role our primitive rules.

\subsection{Definitions}

$\mathbf{0}$ and $\mathbf{1}$ are our step-markers. A marked formula (hereafter an $\mathrm{m}$-formula) is a symbol of the form $\mathbf{0} \varphi$ or $\mathbf{1} \varphi$ for $\varphi \in F m l$.

For $\Delta \subseteq F m l$ and $\boldsymbol{m}=\mathbf{0}$ or $\mathbf{1}$ let $\boldsymbol{m} \Delta=\{\boldsymbol{m} \varphi \mid \varphi \in \Delta\}$. Let $M F m l=$ the set of m-formulas.

\subsection{Heuristic Remark}

In $\boldsymbol{m} \varphi$, the marker $\boldsymbol{m}$ indicates a "mode of acceptance" of $\varphi$, one whose "cash value" lies in certain roles that $\varphi$ can play in deductions. $\mathbf{0}$ marks acceptance as actually true. It is the only step in play in almost all work in logic and mathematics with which I am acquainted; because of this, that literature takes the "no step" approach; it ignores stepping. 1 indicates acceptance as true at a (modally) accessible possible world (or, if you prefer, state), ${ }^{5}$ one can think of $\mathbf{1}$ as indicating one step taken from the "world of evaluation" along an accessibility relation. ${ }^{6}$

When I started work on this paper, I thought of step-markers as indicating mood for deductions: the 0-step as indicative mood, the 1-step as subjunctive mood. I no longer think that this analogy is helpful; for more on this, see 3.10 below.

\section{Model-Theoretic Semantics}

Although the main point of this paper is proof-theoretic, the deductive system will be easier to understand if one has seen how the corresponding model-theory works.

\subsection{Definitions}

Following [7], let $F=\langle W, R, \sqsubseteq\rangle$ be an IK-frame iff: $R \subseteq W^{2}$, $\subseteq W^{2}$ is transitive and reflexive on $W$, and the following conditions are satisfied: "right completeness") for any $u, u^{\prime}$ and $v$, if $u \sqsubseteq u^{\prime}$ and $u R v$ then there is a $v^{\prime}$ so that $v \sqsubseteq v^{\prime}$ and $u^{\prime} R v^{\prime}$; ("left completeness") for any $u, v$ and $v^{\prime}$, if $u R v \sqsubseteq v^{\prime}$ then there is a

\footnotetext{
${ }^{4}$ If for a given logic there is a unique set of natural-deduction rules governing its logical constants that captures the senses of these constants, and thus that carves logical reality at its joints, then the choice of primitive rules should not involve arbitrary choices.

${ }^{5}$ For a different use of markers for formulas, see [2] (p. 172).

${ }^{6} \mathrm{~A}$ caveat: under a reflexive accessibility relation, one can take one step from the actual world and remain where one started.
} 
$u^{\prime}$ so that $u \sqsubseteq u^{\prime} R v^{\prime} . R$ corresponds to modal accessibility as in familiar framemodels for "no step" modal languages; $\sqsubseteq$ corresponds to cognitive accessibility as in familiar frame-models for intuitionistic and intermediate logics. In the following diagram-completion pictures, writing $x$ above $y$ indicates that $y \sqsubseteq x$, horizontal arrows represent $R$, and underlining indicates the existentially bound variable.

$$
\begin{array}{ll}
u^{\prime}--->\frac{v^{\prime}}{u} & \underline{u^{\prime}}--->v^{\prime} \\
u->v & u->v
\end{array}
$$

right completeness left completeness

Consider a IK-frame $F$ as above. Let $W^{F}=W$.

Let $u$ be a dead-end under $F$ iff there is no $v$ so that $u R v$. Let $u R^{+} w$ iff for some $v u \sqsubseteq v$ and $v R w$.

Let $\mathcal{V}$ be a valuation on $W \times S$ iff $\mathcal{V}: W \times S \rightarrow 2$, and $\mathcal{V}$ is persistent with respect to $\sqsubseteq$ (i.e. for any $u, v \in W$ and $\varphi \in S$, if $u \sqsubseteq v$ then $\mathcal{V}(u, \varphi) \leq \mathcal{V}(v, \varphi)$ ).

$\mathcal{M}=\langle F, \mathcal{V}\rangle$ is a model with signature $S$ iff $F$ is a frame and $\mathcal{V}$ is a valuation on $W \times S$. Set $W^{\mathcal{M}}=W^{F}$.

$\mathcal{M}$ is an IK-model iff it is a model and $F$ is an IK-frame.

\subsection{Definitions}

Consider any model $\mathcal{M}$ as above.

For $u \in W^{\mathcal{M}},\langle\mathcal{M}, u\rangle$ is a pointed model. (I will omit brackets where confusion is unlikely.)

We define the relation $=$ between pointed models and formulas by clauses most of which are familiar, but these deserve to be stated:

$\mathcal{M}, u \models(\varphi \supset \psi)$ iff: for any $v$, if $u \sqsubseteq v$ and $\mathcal{M}, v \models \varphi$ then $\mathcal{M}, v \models \psi$;

$\mathcal{M}, u \models \square \varphi$ iff: for any $u$ and $w$, if $u R^{+} w$ then $\mathcal{M}, w \models \varphi$;

$\mathcal{M}, u \models \nabla \varphi$ iff: for some $v, u R v$ and $\mathcal{M}, v \models \varphi$.

As usual, for $\Delta \subseteq F m l$ let $\mathcal{M}, u \models \Delta$ iff for every $\delta \in \Delta \mathcal{M}, u \models \delta$.

\subsection{Persistence Lemma}

Consider an IK-model $\mathcal{M}$. (1) For every $\varphi \in F m l$, if $\mathcal{M}, u \models \varphi$ and $u \sqsubseteq u^{\prime}$ then $\mathcal{M}, u^{\prime} \models \varphi$.

Proof: induction on the stages of the inductive definition of $\mathrm{Fml}$. In the induction step, if $\varphi$ is $\square \psi$, use the transitivity of $\sqsubseteq$. If $\varphi$ is $\diamond \psi$, use the "right completeness" condition.

\subsection{Definitions}

Consider a model $\mathcal{M}=\langle F, \mathcal{V}\rangle$ and $u, v \in W, \varphi \in F m l$, and $\Gamma \subseteq M F m l$.

$\mathcal{M}, u \Vdash \boldsymbol{m} \varphi$ iff $\boldsymbol{m}=\mathbf{0}$, and $\mathcal{M}, u \models \varphi$.

$\mathcal{M}, u, v \Vdash \boldsymbol{m} \varphi$ iff $u R v$, and either $\boldsymbol{m}=\mathbf{0}$ and $\mathcal{M}, u \models \varphi$, or $\boldsymbol{m}=\mathbf{1}$ and $\mathcal{M}, v \models \varphi$.

$\mathcal{M}, u \Vdash \Gamma$ iff for every $\gamma \in \Gamma \mathcal{M}, u \Vdash \gamma$.

$\mathcal{M}, u, v \Vdash \Gamma$ iff $u R v$, and for every $\gamma \in \Gamma \mathcal{M}, u, v \Vdash \gamma$. 


\subsection{Heuristic Remark}

Think of $\mathbf{1}$ as carrying a free variable that relative to $u$ ranges over $\{v \mid u R v\}$. Since that set can be empty, our proof-theoretic systems will bear a structural similarity to systems tailored to free-logic semantics.

\subsection{Definitions}

Let $\langle\Gamma, \chi\rangle$ be an inference iff $\Gamma \subseteq M F m l$ and $\chi \in M F m l$.

Consider an inference $\langle\Gamma, \chi\rangle$. Given a model $\mathcal{M}$ and $u \in W^{\mathcal{M}},\langle\Gamma, \chi\rangle$ is $\mathcal{M}$ valid at $u$ iff: (V1) if $u$ is a dead-end and $\mathcal{M}, u \Vdash \Gamma$ (in which case $\Gamma \subseteq \mathbf{0} F m l$ ), then $\mathcal{M}, u \Vdash \chi$ (in which case $\chi \in \mathbf{0} F m l$ ); (V2) for every $v$, if $\mathcal{M}, u, v \models \Gamma$ then $\mathcal{M}, u, v=\chi \cdot\langle\Gamma, \chi\rangle$ is $\mathcal{M}$-valid iff it is is $\mathcal{M}$-valid at every $u \in W^{\mathcal{M}}$.

A weaker property deserves some attention: let $\langle\Gamma, \chi\rangle$ is $\mathcal{M}$-valid ${ }^{-}$at $u$ iff: $\left(\mathrm{V}^{-}{ }^{-}\right)$ if $\mathcal{M}, u \Vdash \Gamma$ then $\mathcal{M}, u \Vdash \chi$; (V2) is true. $\langle\Gamma, \chi\rangle$ is $\mathcal{M}$-valid ${ }^{-}$iff it is is $\mathcal{M}$-valid ${ }^{-}$ at every $u \in W^{\mathcal{M}}$.

$\langle\Gamma, \chi\rangle$ is IK-valid [IK-valid ${ }^{-}$], alternatively $\chi$ is an IK-consequence [IKconsequence $^{-}$] of $\Gamma$, iff for every IK-model $\mathcal{M},\langle\Gamma, \chi\rangle$ is $\mathcal{M}$-valid [ $\mathcal{M}$-valid $^{-}$].

For $\chi \in M F m l, \chi$ is $\mathcal{M}$-valid [ $\mathcal{M}$-valid $\left.{ }^{-}\right]$iff $\langle\{\}, \chi\rangle$ is $\mathcal{M}$-valid [ $\mathcal{M}$-valid ${ }^{-}$].

$\chi$ is IK-valid [IK-valid ${ }^{-}$] iff for every IK-model $\mathcal{M}, \varphi$ is $\mathcal{M}$-valid [ $\mathcal{M}$-valid $\left.{ }^{-}\right]$.

\subsection{Consider any Model $\mathcal{M}$}

\subsubsection{Observations}

Consider any $u \in W^{\mathcal{M}}$ and $\theta \in F m l$. (1) $\langle\Gamma, \mathbf{0} \theta\rangle$ is $\mathcal{M}$-valid $^{-}$at $u$ iff it is $\mathcal{M}$-valid at $u$. (2) If $\langle\Gamma, \mathbf{1} \theta\rangle$ is $\mathcal{M}$-valid $^{-}$at $u$ then it is $\mathcal{M}$-valid at $u$. (3) $\langle\{\mathbf{0} \diamond \top\}, \mathbf{1} \top\rangle$ is $\mathcal{M}$ valid $^{-}$at $u$ iff $u$ is a dead-end in $W^{\mathcal{M}}$. These observations show that for inferences IK-validity is slightly stronger than IK-validity ${ }^{-}$.

Proofs. For (1), left-to-right is straightforward. Assume the right-side. Assume that $\mathcal{M}, u \Vdash \Gamma$. If $u$ is a dead-end in $W^{\mathcal{M}}, \mathcal{M}, u \Vdash \mathbf{0} \theta$. If $u$ is not a dead-end, fix a $v$ so that $u R v$; so $\mathcal{M}, u, v \Vdash \Gamma$; so $\mathcal{M}, u, v \Vdash \mathbf{0} \theta$; so $\mathcal{M}, u \Vdash \mathbf{0} \theta$. The left-side follows. (2) is straightforward. For (3), assume the right-side. So $\mathcal{M}, u \nVdash \mathbf{0} \diamond \top$; the left-side vacuously follows. Assume the leftside. Assume that $u$ is not a dead-end. So $\mathcal{M}, u \models \diamond \top$; so $\mathcal{M}, u \Vdash \mathbf{0} \diamond \top$. By the left-side, $\mathcal{M}, u \Vdash \mathbf{1} \top$; so $\mathbf{1} \top \in \mathbf{0 F m l}$, a contradiction. The right-side follows.

\subsubsection{Observations}

(1) If $\langle\Gamma, \mathbf{1} \theta\rangle$ is $\mathcal{M}$-valid and every member of $W^{\mathcal{M}}$ is a dead-end, then $\langle\Gamma, \mathbf{1} \theta\rangle$ is $\mathcal{M}$-valid $^{-}$. (2) $\langle\{\boldsymbol{0} \diamond \top\}, \mathbf{1} T\rangle$ is $\mathcal{M}$-valid, and so is IK-valid. (3) If some member of $W^{\mathcal{M}}$ is not a dead-end then $\langle\{\mathbf{0} \diamond \top\}, \mathbf{1} \top\rangle$ is not $\mathcal{M}$-valid $^{-}$. (4) For any $\Sigma \subseteq F m l$ and $\theta \in F m l$, if $\langle\mathbf{0} \Sigma, \mathbf{1} \theta\rangle$ is $\mathcal{M}$-valid ${ }^{-}$then so is $\langle\mathbf{0} \Sigma, \mathbf{0} \perp\rangle$. 
Proofs. For (1), assume the if-clause. Consider any $u \in W^{\mathcal{M}}$. Assume that $\mathcal{M}, u \Vdash \Gamma$; so $\mathcal{M}, u \Vdash \mathbf{1} \theta$, a contradiction; so $\langle\Gamma, \mathbf{1} \theta\rangle$ vacuously satisfies (V1). For any $v, \mathcal{M}, u, v \nVdash \Gamma$ because not $u R v$; so $\langle\Gamma, \mathbf{1} \theta\rangle$ vacuously satisfies (V2), yielding the then-clause. For (2): if $u$ is a dead-end in $W^{\mathcal{M}}$ then $\mathcal{M}, u \nVdash \mathbf{0} \diamond \top$, showing (V1) to be vacuously true of $\langle\{\mathbf{0} \diamond \top\}, \mathbf{1} \top\rangle$. If $u$ is not a dead-end, (V2) is true of $\langle\{\mathbf{0} \diamond \top\}, \mathbf{1} T\rangle$. Universally quantifying-out $u$, and then $\mathcal{M}$, (2) follows. (3) follows from 2.7.1.(3). Assume that $\langle\mathbf{0} \Sigma, \mathbf{1} \theta\rangle$ is $\mathcal{M}$-valid $^{-}$. For any $v$, if $\mathcal{M}, u, v \Vdash \mathbf{0} \Sigma$ then $\mathcal{M}, u \Vdash \mathbf{0} \Sigma$; so by $\left(\mathrm{V}^{-}\right) \mathcal{M}, u \Vdash \mathbf{1} \theta$; so $\mathbf{1} \theta \in \mathbf{0} F m l$, a contradiction. So $\mathcal{M}, u, v \nVdash \mathbf{0} \Sigma$ and $\mathcal{M}, u \nVdash \mathbf{0} \Sigma$. So $\langle\mathbf{0} \Sigma, \mathbf{0} \perp\rangle$ is vacuously $\mathcal{M}$-valid ${ }^{-}$, proving (4).

\subsubsection{Observations}

(1) If $\chi$ is $\mathcal{M}$-valid ${ }^{-}$then $\chi \in \mathbf{0} F m l$. (2) If $\chi$ is $\mathcal{M}$-valid and $W^{\mathcal{M}}$ contains a deadend then $\chi \in \mathbf{0 F m l}$. (3) $\mathbf{1} \top$ is $\mathcal{M}$-valid iff $W^{\mathcal{M}}$ contains no dead-end. (4) $\chi$ is IK-valid $^{-}$iff $\chi$ is IK-valid.

Proofs. (1) is straightforward. Assume (2)'s if-clause; fix $u$ to be a dead-end in $W^{\mathcal{M}}$. Since $\mathcal{M}, u \Vdash \chi, \chi \in \mathbf{0} F m l$, yielding (2). Assume that $\mathbf{1} \top$ is $\mathcal{M}$-valid. If $W^{\mathcal{M}}$ contains a dead-end, (2) yields a contradiction. Assume (3)'s right-side. For any $u \in W^{\mathcal{M}}$, fix a $v$ so that $u R v$; so $\mathcal{M}, u, v \Vdash 1 \top$. So $1 \top$ is $\mathcal{M}$-valid. (3) follows. Assume that $\chi$ is IK-valid ${ }^{-}$. By (1) $\chi \in \mathbf{0 F m l}$; so by 2.7.1.(1) $\chi$ is IK-valid. Assume that $\chi$ is IK-valid. Since there are models whose frames contain dead-ends, by 2.7.3.(2) $\chi \in \mathbf{0 F m l}$; so by 2.7.1.(1) $\chi$ is IK-valid $^{-}$. (4) follows.

\subsection{Observation}

$\mathbf{0} \perp$ and $\mathbf{1} \perp$ are IK-equivalent in this sense: $\langle\{\mathbf{0} \perp\}, \mathbf{1} \perp\rangle$ and $\langle\{\mathbf{1} \perp\}, \mathbf{0} \perp\rangle$ are IK-valid. So in a sense $\mathbf{0} \perp$ and $\mathbf{1} \perp$ are degenerate marked-formulas, since the markers "don't matter". 7

\section{The Proof-Theoretic System IK}

\subsection{Definitions}

A string is a function with domain in $\omega$. Let ${ }^{\wedge}$ be concatenation for strings. For any string $s_{1}, s_{0} \preceq s_{1}$ iff for some string $s s_{1}=s_{0} \wedge$. Read $\preceq$ as "is an initial segment of".

$T$ is a naked (aka bare) tree iff $T$ is a non-empty set of strings of natural numbers closed under taking initial segments (i.e. for any $s \in T$, if $t \preceq s$ then $t \in T$ ) and $T$ satisfies the "no left gaps" condition (i.e. for any $s, m$ and $n$, if $s^{\wedge}[m] \in T$ and $n<m$ then $\left.s^{\wedge}[n] \in T\right)$. $s$ is a leaf of a naked tree $T$ iff $s \in T$ and for every $s^{\prime}$, if $s^{\prime} \in T$ and $s \preceq s^{\prime}$ then $s=s^{\prime}$. For such a $T$ and $s \in T$, let $T_{s}=\left\{t \mid s^{\wedge} t \in T\right\}$.

\footnotetext{
${ }^{7}$ Had we allowed for "exploding" IK-models instead of our "non-exploding" ones in 2.6, we would have a narrower IK-consequence relation; e.g. $\langle\{\mathbf{1} \perp\}, \mathbf{0} \perp\rangle$ would not be IK-valid. This is not the place for a full treatment of exploding models.
} 


\subsection{Remarks}

[ ] is a member, the "root", of every naked tree. So $\{[]\}$ is the smallest naked tree. The "no left gaps" condition insures that any two naked trees are identical if they are isomorphic with respect to $\preceq{ }^{8}$

\subsection{Definition}

A labeled tree is a function whose domain is a naked tree. For a labeled tree $\mathcal{T}$, let $\mathcal{T}_{s}$ be the labeled tree with domain $\operatorname{dom}(\mathcal{T})_{s}$ and such that $\mathcal{T}_{s}(t)=\mathcal{T}\left(s^{\wedge} t\right)$ for each $t \in \operatorname{dom}(\mathcal{T})_{s}$

\subsection{Notation}

For the rest of this paper, we will make use of a countably infinite set Var of variables (disjoint from the other sets just introduced, and neither step-markers is a variable).

Definition. A tagged $\mathrm{m}$-formula (i.e. a primitive type-assignment) is a symbol of the form $v: \chi$ for $\chi \in M F m l$; $v$ is its tag. We will sometimes treat $v: \chi$ as if it were an ordered pair.

\subsection{Definitions}

$C$ is a context iff $C$ is a single-valued set of tagged m-formulas (i.e. for any $v, \chi$ and $\chi^{\prime}$, if $v: \chi, v: \chi^{\prime} \in C$ then $\chi$ is $\left.\chi^{\prime}\right)$. So $\operatorname{dom}(C)=$ the set of variables occurring on the left-side of members of $C$, and $\operatorname{ran}(C)=$ the set of $m$-formulas occurring on the right-side of members of $C$. A set of contexts is coherent iff its union is a context.

\subsection{Preparatory Remarks}

In 3.8 below we will inductively define a type-assignment system $\Rightarrow_{I K}$. Informally, " $C \Rightarrow_{I K} \mathcal{D}: \chi$ " means: relative to context $C, \mathcal{D}$ is an IK-deduction with conclusion $\chi$. Through Section 7, ' $\Rightarrow$ ' and 'deduction' abbreviate ' $\Rightarrow_{I K}$ ' and 'IK-deduction'.

As is usual, in " $C \Rightarrow \mathcal{D}: \chi$ " the mention of $\chi$ is redundant, since $\mathcal{D}$ will uniquely determine $\chi$. Our definition will be slightly unusual because it will require that if $C \Rightarrow \mathcal{D}: \chi$ and $C^{\prime} \Rightarrow \mathcal{D}: \chi$ then $C=C^{\prime}$; the idea is to keep contexts relative to which labeled trees are deductions as small as possible.

A deduction will be a labeled tree. Leaves of $\operatorname{dom}(\mathcal{D})$ will be labeled by tagged $\mathrm{m}$ formulas; non-leaves in $\operatorname{dom}(\mathcal{D})$ will be labeled by either an $\mathrm{m}$-formula or an ordered pair of an $\mathrm{m}$-formula followed by a tagged $\mathrm{m}$-formula or an ordered triple of an $\mathrm{m}$ formula followed by two tagged m-formulas. In the latter two cases, the left-most

\footnotetext{
${ }^{8}$ The following multi-place operation on naked trees will briefly be convenient.

Definition. For $n \in \omega, n>0$ and naked trees $T_{i \in(n)}$, let

$$
\#\left(T_{1}, \ldots, T_{n}\right)=\{[]\} \cup\left\{[i]^{\wedge} s \mid s \in T_{i}, i \in(n)\right\} .
$$
}


component is the $\mathrm{m}$-formula label, and the other components indicate discharging of those tagged $\mathrm{m}$-formulas at that string.

In what follows, distinct metavariables (e.g. ' $\nu_{0}$ ' and ' $\nu_{1}$ ') represent distinct variables. Let " $C, \nu: \xi \Rightarrow \mathcal{D}: \chi$ " abbreviate this: $\nu \notin \operatorname{dom}(C)$, and either $C \cup\{\nu: \xi\} \Rightarrow$ $\mathcal{D}: \chi$ or $C \Rightarrow \mathcal{D}: \chi$. So that notation DOES NOT indicate that $\nu: \xi$ belongs to the context relative to which $\mathcal{D}$ is a deduction! Extend this notation in the obvious way to allow for $C, v_{1}: \xi_{1}, \ldots, v_{k}: \xi_{k} \Rightarrow \mathcal{D}: \chi$.

The induction clauses will be presented pictorially. Discharging of an occurrence of a tagged formula (or equivalently of the leaf it labels) will be indicated by putting that label within square-brackets. The discharge at a given string in $\operatorname{dom}(\mathcal{D})$ of some tagged $\mathrm{m}$-formula(s) will be indicated by superscripting the relevant tagged $\mathrm{m}$ formula(s) being discharged, though to minimize clutter in practice, if the discharging is non-vacuous it is convenient to write only the tagging variable(s).

We will define the "dependency-set" $d p d(\mathcal{D})$ for $\mathcal{D}$, informally the set of leaves of $\operatorname{dom}(\mathcal{D})$ on which $\mathcal{D}$ "depends", simultaneously with $\Rightarrow$.

\subsection{Definitions}

For $s \in \operatorname{dom}(\mathcal{D})$, let $s$ be open in $\mathcal{D}$ iff for every $s^{\prime} \in \operatorname{dpd}\left(\mathcal{D}_{s}\right), s^{\wedge} s^{\prime} \in d p d(\mathcal{D}) .{ }^{9}$

Consider a context $C$. A barrier in $\mathcal{D}$ with with exception for $C$ will be an antichain $\left\{s_{1}, \ldots, s_{m}\right\} \subseteq \operatorname{dom}(\mathcal{D})$ such that (i) for each $i \in(m) s_{i}$ is open in $\mathcal{D}$, the formula-label of $s_{i}$ in $\mathcal{D}$ is in $\mathbf{0 F m l}$, there is no $t \in d p d(\mathcal{D})$ with $s_{i} \preceq t$ and $\mathcal{D}(t) \in$ $C$, but (the non-vacuity condition) for some $t \in d p d(\mathcal{D})$ with $s_{i} \preceq t$ there is a $v$ and $\varphi$ such that $\mathcal{D}(t)=v: \mathbf{1} \varphi$, and (ii) for every $t \in d p d(\mathcal{D})$ with $\mathcal{D}(t)$ of the form $v: \mathbf{1} \varphi$ for $v \notin \operatorname{dom}(C)$ there is an $i \in(m)$ with $s_{i} \preceq t{ }^{10}$ If $C=\{\mu: \mathbf{1} \psi\}$, let $\left\{s_{1}, \ldots, s_{m}\right\}$ be a barrier with exception for $\mu: \mathbf{1} \psi$ iff it is a barrier with exception for $C$.

\subsection{Definition}

Now, our much awaited definition of $\Rightarrow$. It has two base-clauses.

Assumption For $v \in V$ ar and $\chi \in M F m l, v: \chi \Rightarrow \mathcal{D}: \chi$ for $\mathcal{D}=\{\langle[], v: \chi\rangle\}$, i.e. $\mathcal{D}$ is [ ] labelled by $v: \chi$. Also $d p d(\mathcal{D})=\operatorname{dom}(\mathcal{D})=\{[]\}$.

$\mathbf{0} \top$ Introduction $\quad\{\} \Rightarrow \mathcal{D}: \mathbf{0} \top$ for $\mathcal{D}=\{\langle[], \mathbf{0} \top\rangle\} ; \mathcal{D}$ may be pictured thus.

$$
\overline{\mathbf{0}}^{\mathbf{0}} \boldsymbol{\top} I
$$

Also $\operatorname{dpd}(\mathcal{D})=\{[]\}$.The over-bar indicates that $\mathbf{0} \top$ is not an assumption in $\mathcal{D}$; one might think of it as being inferred from the empty set of assumptions.

Next, the induction clauses, starting with $\mathbf{0}$-versions of all but one of the standard rules for the Boolean constants.

\footnotetext{
${ }^{9}$ Informally: for every leaf of $\mathcal{D}$ identical to or above $s$, if it was not discharged by the time we reach $s$ (reading down from the leaves), then it is not discharged in $\mathcal{D}$.

${ }^{10}$ Informally; members of a barrier "isolate" leaves whose m-formula labels are marked by $\mathbf{1}$, other than labels in the exception set. Nothing precludes a barrier from being empty.
} 
$\mathbf{0} \perp$ Elimination For $\chi \in M F m l$, if $C \Rightarrow \mathcal{D}_{0}: \mathbf{0} \perp$ then $C \Rightarrow \mathcal{D}: \chi$ for $\mathcal{D}$ as pictured. $^{11}$

$$
\begin{gathered}
\mathcal{D}_{0} \\
\frac{\mathbf{0} \perp}{\chi} \mathbf{0} \perp E
\end{gathered}
$$

Also, $\operatorname{dpd}(\mathcal{D})=\left\{[0]^{\wedge} s \mid s \in \operatorname{dpd}\left(\mathcal{D}_{0}\right)\right\}$.

0 $\supset$ Introduction If $C, v: \mathbf{0} \varphi \Rightarrow \mathcal{D}_{0}: \mathbf{0} \psi$ then $C \Rightarrow \mathcal{D}: \mathbf{0}(\psi \supset \varphi)$ for $\mathcal{D}$ as pictured.

$$
\begin{gathered}
{[v: \mathbf{0} \varphi]} \\
\mathcal{D}_{0} \\
\mathbf{0} \psi \\
\mathbf{0}(\varphi \supset \psi)^{v}
\end{gathered}
$$

Let $\operatorname{dpd}(\mathcal{D})=\left\{[0]^{\wedge} s \mid s \in \operatorname{dpd}\left(\mathcal{D}_{0}\right)\right.$ and $\left.\mathcal{D}_{0}(s) \neq v: \mathbf{0} \varphi\right\}$.

$\mathbf{0} \vee$ Introduction For $i \in 2$, if $C \Rightarrow \mathcal{D}_{0}: \mathbf{0} \varphi_{i}$ then $C \Rightarrow \mathcal{D}: \mathbf{0}\left(\varphi_{0} \vee \varphi_{1}\right)$ for $\mathcal{D}$ as pictured.

Let $\operatorname{dpd}(\mathcal{D})=\left\{[0]^{\wedge} s \mid s \in \operatorname{dpd}\left(\mathcal{D}_{0}\right)\right\}$.

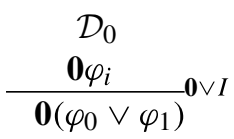

$\mathbf{0} \vee$ Elimination If $C_{2} \Rightarrow \mathcal{D}_{2}: \mathbf{0}\left(\varphi_{0} \vee \varphi_{1}\right)$, for both $i \in 2 C_{i}, v_{i}: \mathbf{0} \varphi_{i} \Rightarrow \mathcal{D}_{i}: \chi, v_{0}, v_{1} \in$ Var are distinct, and $\left\{C_{0}, C_{1}, C_{2}\right\}$ is coherent, then $\bigcup_{i \in 3} C_{i} \Rightarrow \mathcal{D}: \chi$ for $\mathcal{D}$ as pictured.

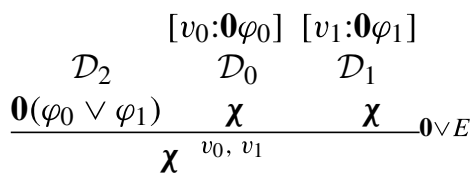

Let $\operatorname{dpd}(\mathcal{D})=\bigcup_{i \in 2}\left\{[i+1]^{\wedge} s \mid s \in \operatorname{dpd}\left(\mathcal{D}_{i}\right), \mathcal{D}_{i}(s) \neq v_{i}: 0 \varphi_{i}\right\} \cup\left\{[0]^{\wedge} s \mid s \in\right.$ $\left.\operatorname{dpd}\left(\mathcal{D}_{2}\right)\right\} .^{12}$

0\& Introduction If for $i \in\{2\}, C_{i} \Rightarrow \mathcal{D}_{i}: \mathbf{0} \varphi_{i}$ and $\left\{C_{0}, C_{1}\right\}$ is coherent, then $C_{0} \cup$ $C_{1} \Rightarrow \mathcal{D}: \mathbf{0}\left(\varphi_{0} \& \varphi_{1}\right)$ for $\mathcal{D}$ as pictured. ${ }^{13}$

$$
\begin{array}{cc}
\mathcal{D}_{0} & \mathcal{D}_{1} \\
\mathbf{0} \varphi_{0} & \mathbf{0} \varphi_{1} \\
\hline \boldsymbol{0}\left(\varphi_{0} \& \varphi_{1}\right)
\end{array} \mathbf{0} I
$$

Let $d p d(\mathcal{D})=\bigcup_{i \in 2}\left\{[i]^{\wedge} s \mid s \in d p d\left(\mathcal{D}_{i}\right)\right\}$.

0\& Elimination For $i \in 2$, if $C \Rightarrow \mathcal{D}_{0}: \mathbf{0}\left(\varphi_{0} \& \varphi_{1}\right)$, then $C \Rightarrow \mathcal{D}: \mathbf{0} \varphi_{i}$ for $\mathcal{D}$ as pictured.

Let $\operatorname{dpd}(\mathcal{D})=\left\{[0]^{\wedge} s \mid s \in \operatorname{dpd}\left(\mathcal{D}_{0}\right)\right\}$.

$$
\begin{gathered}
\mathcal{D}_{0} \\
\frac{\mathbf{0}\left(\varphi_{0} \& \varphi_{1}\right)}{\mathbf{0} \varphi_{i}} \mathbf{0} \& E
\end{gathered}
$$

\footnotetext{
${ }^{11}$ An interpretive aid: $\operatorname{dom}(\mathcal{D})=\#\left(\mathcal{D}_{0}\right)$.

${ }^{12}$ Another interpretive aid: $\operatorname{dom}(\mathcal{D})=\#\left(\mathcal{D}_{2}, \mathcal{D}_{0}, \mathcal{D}_{1}\right)$.

${ }^{13}$ Another interpretive aid: $\operatorname{dom}(\mathcal{D})=\#\left(\mathcal{D}_{1}, \mathcal{D}_{0}\right)$. Hopefully from now on the reader will not need these aids.
} 
The next rule is really two rules, one for each choice of marker $\boldsymbol{m}$.

$\boldsymbol{m} \supset$ Elimination If $C_{1} \Rightarrow \mathcal{D}_{1}: \boldsymbol{m}(\psi \supset \varphi), C_{0} \Rightarrow \mathcal{D}_{0}: \boldsymbol{m} \psi$, and $\left\{C_{0}, C_{1}\right\}$ is coherent, then $C_{0} \cup C_{1} \Rightarrow \mathcal{D}: m \varphi$ for $\mathcal{D}$ as pictured.

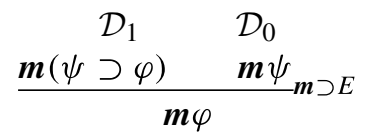

Let $\operatorname{dpd}(\mathcal{D})=\bigcup_{i \in 2}\left\{[1-i]^{\wedge} s \mid s \in \operatorname{dom}\left(\mathcal{D}_{i}\right)\right\}$.

The next two rules have principal formulas marked by $\mathbf{1}{ }^{14}$

$\mathbf{1} \perp$ Elimination, For $\theta \in F m l$, if $C \Rightarrow \mathcal{D}_{0}: \mathbf{1} \perp$ then $C \Rightarrow \mathcal{D}: \mathbf{0} \theta$ for $\mathcal{D}$ as pictured.

$$
\begin{aligned}
& \mathcal{D}_{0} \\
& \frac{\mathbf{1} \perp}{\mathbf{0} \theta} \mathbf{1} \perp E_{\mathbf{0}}
\end{aligned}
$$

Also, $\operatorname{dpd}(\mathcal{D})=\left\{[0]^{\wedge} s \mid s \in d p d\left(\mathcal{D}_{i}\right)\right\} .^{15}$

$\mathbf{1} \vee$ Elimination 0 If $C_{2} \Rightarrow \mathcal{D}_{2}: \mathbf{1}\left(\varphi_{0} \vee \varphi_{1}\right)$, for both $i \in 2 C_{i}, v_{i}: \mathbf{1} \varphi_{i} \Rightarrow \mathcal{D}_{i}: \mathbf{0} \theta$, $\nu_{0}, \nu_{1} \in V a r$ are distinct, and $\left\{C_{0}, C_{1}, C_{2}\right\}$ is coherent, then $\bigcup_{i \in 3} C_{i} \Rightarrow \mathcal{D}: \mathbf{0} \theta$ for $\mathcal{D}$ as pictured.

$\left.\begin{array}{ccc}\multicolumn{4}{c}{\left[v_{0}: \mathbf{1} \varphi_{0}\right]} & {\left[v_{1}: \mathbf{1} \varphi_{1}\right]} \\ \mathcal{D}_{2} & \mathcal{D}_{0} & \mathcal{D}_{1} \\ \mathbf{1}\left(\varphi_{0} \vee \varphi_{1}\right) & \mathbf{0} \theta & \mathbf{0} \theta\end{array}\right] \mathbf{1} \vee E_{\mathbf{0}}$

Let $\operatorname{dpd}(\mathcal{D})=\bigcup_{i \in 2}\left\{[i+1]^{\wedge} s \mid s \in \operatorname{dpd}\left(\mathcal{D}_{i}\right), \mathcal{D}_{i}(s) \neq v_{i}: \mathbf{1} \varphi_{i}\right\} \cup\left\{[0]^{\wedge} s \mid s \in\right.$ $\left.\operatorname{dpd}\left(\mathcal{D}_{2}\right)\right\}$. Note that this rule, unlike $\mathbf{0} \vee E$, requires that its conclusion be marked by $\mathbf{0}$. But see $1 \vee E_{1}$ in the next section.

The next rule differs from its $\mathbf{0}$-cousin by requiring a minor premise.

$1 \supset$ Introduction If $C_{0} \cup\{v: \mathbf{1} \varphi\} \Rightarrow \mathcal{D}_{0}: \mathbf{1} \psi, C_{1} \Rightarrow \mathcal{D}_{1}: \mathbf{1} \theta$, and $\left\{C_{0}, C_{1}\right\}$ is coherent, then $C_{0} \cup C_{1} \Rightarrow \mathcal{D}: \mathbf{1}(\varphi \supset \psi)$ for $\mathcal{D}$ as pictured.

$$
\begin{aligned}
& {[v: \mathbf{1} \varphi]} \\
& \mathcal{D}_{0} \quad \mathcal{D}_{1} \\
& \frac{\mathbf{1} \psi}{\mathbf{1}(\varphi \supset \psi)^{v}}{ }^{v I}
\end{aligned}
$$

Let $\operatorname{dpd}(\mathcal{D})=\left\{[0]^{\wedge} s \mid s \in \operatorname{dpd}\left(\mathcal{D}_{0}\right), \mathcal{D}_{0}(s) \neq v: \mathbf{1} \varphi\right\} \cup\left\{[1]^{\wedge} s \mid s \in \operatorname{dpd}\left(\mathcal{D}_{1}\right)\right\}$.

The next rule is the only pure step-rule of IK.

\footnotetext{
${ }^{14}$ The reader may wonder why the conclusions for $\mathbf{1}$-versions of $\perp E$ and $\vee E$ are marked only by $\mathbf{0}$. The point is to minimize the class of instances of primitive rules; we lose nothing by restricting these rules to conclusions in $\mathbf{0} \mathrm{Fml}$.

${ }^{15}$ Along with $\mathbf{0} \perp E$, this rule makes $\mathbf{0} \perp$ and $\mathbf{1} \perp$ interdeducible. Were we to work with exploding IKmodels, we would drop this rule.
} 
Transfer $_{0} \quad$ If \{\}$\Rightarrow \mathcal{D}_{0}: \mathbf{0} \varphi$ and $C \Rightarrow \mathcal{D}_{1}: \mathbf{1} \theta$, then $C \Rightarrow \mathcal{D}: \mathbf{1} \varphi$ for $\mathcal{D}$ as pictured.

$$
\begin{array}{ll}
\mathcal{D}_{0} & \mathcal{D}_{1} \\
\mathbf{0} \varphi & \mathbf{1} \theta
\end{array} \operatorname{Tr}_{0}
$$

Let $\operatorname{dpd}(\mathcal{D})=\bigcup_{i \in 2}\left\{[i]^{\wedge} s \mid s \in \operatorname{dpd}\left(\mathcal{D}_{i}\right)\right\}$.

The introduction rules for $\square$ and $\diamond$ will "freeze" a formula marked by $\mathbf{1}$ into a modal formula marked by $\mathbf{0}$; the elimination rules reverse this freezing.

$\diamond$ Introduction $\quad$ If $C \Rightarrow \mathcal{D}_{0}: \mathbf{1} \varphi$, then $C \Rightarrow \mathcal{D}: \mathbf{0} \diamond \varphi$ for this $\mathcal{D}$.

$$
\begin{aligned}
& \mathcal{D}_{0} \\
& \frac{\mathbf{1} \varphi}{\mathbf{0} \triangleright \varphi} \diamond I
\end{aligned}
$$

Let $\operatorname{dpd}(\mathcal{D})=\left\{[0]^{\wedge} s \mid s \in \operatorname{dpd}\left(\mathcal{D}_{0}\right)\right\}$.

$\diamond$ Elimination If $C_{0}, v: \mathbf{1} \varphi \Rightarrow \mathcal{D}_{0}: \mathbf{0} \psi, \mathcal{D}_{0}$ has a barrier with exception for $v: \mathbf{1} \varphi$, $C_{1} \Rightarrow \mathcal{D}_{1}: \mathbf{0} \nabla \varphi$, and $\left\{C_{0}, C_{1}\right\}$ is coherent, then $C_{0} \cup C_{1} \Rightarrow \mathcal{D}: \mathbf{0} \psi$ for this $\mathcal{D}$.

$$
\begin{aligned}
& \text { [ } \nu: \mathbf{1} \varphi \text { ] } \\
& \mathcal{D}_{1} \quad \mathcal{D}_{0} \\
& \frac{\mathbf{0} \diamond \varphi \quad \mathbf{0} \psi}{\mathbf{0} \psi^{v}} \diamond E
\end{aligned}
$$

Let $\operatorname{dpd}(\mathcal{D})=\left\{[0]^{\wedge} s \mid s \in \operatorname{dpd}\left(\mathcal{D}_{1}\right)\right\} \cup\left\{[1]^{\wedge} s \mid s \in \operatorname{dpd}\left(\mathcal{D}_{0}\right), \mathcal{D}_{0}(s) \neq v: \mathbf{1} \varphi\right\}$.

$\square$ Introduction If $C, v: \mathbf{1} \top \Rightarrow \mathcal{D}_{0}: \mathbf{1} \varphi$, and $\mathcal{D}_{0}$ has a barrier with exception for $v: \mathbf{1} \top$, then $C \Rightarrow \mathcal{D}: \mathbf{0} \square \varphi$ for this $\mathcal{D}$.

$$
\begin{gathered}
{[\nu: \mathbf{1} \top]} \\
\mathcal{D}_{0} \\
\frac{\mathbf{1} \varphi}{\mathbf{0} \square \varphi^{\nu}} \square I
\end{gathered}
$$

Let $\operatorname{dpd}(\mathcal{D})=\left\{[0]^{\wedge} s \mid s \in \operatorname{dpd}\left(\mathcal{D}_{0}\right), \mathcal{D}_{0}(s) \neq v: \mathbf{1} \top\right\}$.

$\square$ Elimination If $C_{0} \Rightarrow \mathcal{D}_{0}: \mathbf{0} \square \varphi, C_{1} \Rightarrow \mathcal{D}_{1}: \mathbf{1} \theta$, and $\left\{C_{0}, C_{1}\right\}$ is coherent, then $C \Rightarrow \mathcal{D}: \mathbf{1} \varphi$ for this $\mathcal{D}$.

$$
\begin{aligned}
& \mathcal{D}_{0} \quad \mathcal{D}_{1} \\
& \frac{\mathbf{0} \square \varphi \quad \mathbf{1} \theta}{\mathbf{1} \varphi} \square E
\end{aligned}
$$

Let $\operatorname{dpd}(\mathcal{D})=\bigcup_{i \in 2}\left\{[i]^{\wedge} s \mid s \in \operatorname{dpd}\left(\mathcal{D}_{i}\right)\right\}$.

Let $\Rightarrow{ }_{I K}^{-}$be the type-assignment system obtained by stopping here. To finish defining $\Rightarrow_{I K}$ we add one more rule.

$\diamond$ Elimination $^{+} \quad$ If $C \Rightarrow \mathcal{D}_{0}: \mathbf{0} \diamond \top$ then $C \Rightarrow \mathcal{D}: \mathbf{1} \top$ for this $\mathcal{D}$.

$$
\frac{\mathcal{D}_{0}}{\mathbf{0} \triangleright \top}{ }_{\mathbf{1} \top} \nabla E^{+}
$$




\subsection{Remarks}

A well-known point, but perhaps worth repeating: discharging is, in effect, variablebinding: the discharging superscripts bind all the occurrences of the associated variables at the leaves labeled by the discharged tagged formulas.

The barrier-conditions for $\diamond E$ and $\square I$ correspond to the conditions on the eigenvariable for $\exists E$ and $\forall I$ in natural-deduction systems for first-order logic. The motivation for these conditions will be clarified by the soundness lemmas for these rules.

The minor premises for $\mathbf{1} \supset I$ and $\square E$ play a role that resembles the role of the singular-existence premises for $\forall E$ in free first-order natural-deduction systems. This will be made clearer by the soundness lemmas for these rules.

$\diamond E^{+}$differs from the other rules governing $\diamond$ and $\square$ by virtue of having only one instance: from $\mathbf{0} \diamond \top$ to $\mathbf{1} T$. I see no non-ad-hoc way to strengthen $\diamond E$ so as to make $\diamond E^{+}$admissible. $^{16}$

\subsection{Remarks}

When writing an earlier version of this paper, I viewed the occurrence of the marker in the conclusion of a deduction as a "moodal" operator on that deduction: we could rewrite $C \Rightarrow \mathcal{D}: \boldsymbol{m} \varphi$ as $C \Rightarrow \boldsymbol{m}(\mathcal{D}): \varphi$, indicating that relative to $C \boldsymbol{m}(\mathcal{D})$ is a deduction of $\varphi$; its mood is indicative if $\boldsymbol{m}=\mathbf{0}$ and subjunctive if $\boldsymbol{m}=\mathbf{1}$. I was led in this direction by thinking of mood in natural languages (and formal languages intended to reflect features of natural languages) as a matter of embeddable operators on formulas, along the lines formulated by Lloyd Humberstone in [4]. ${ }^{17}$ (As a marker on formulas, $\boldsymbol{m}$ cannot be embedded; but as an operator on deductions, it can - if $\operatorname{dom}(\mathcal{D}) \neq\{[]\}, \mathcal{D}$ contains step-markers above its root.) Work by Kai Wehmeier and Helge Rückert $[9,10]$ persuaded me that mood, as a feature of natural-languages, is best captured in a formal language by markers attaching to predicate-expressions, quantifier-expressions, and modal operators, rather than by operators on formulas. Thus the analogy between mood, properly understood, and step-markers as used in this paper is more misleading than helpful. ${ }^{18}$

${ }^{16}$ The following revision of $\operatorname{Tr} n_{0}$ would render $\diamond E^{+}$admissible.

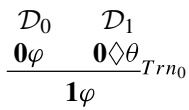

But it is preferable that $\operatorname{Tr} n_{0}$ be a pure one-step rule, and the special accommodation of the inference from $\mathbf{0} \diamond \top$ to $1 \top$ be undisguised.

${ }^{17}$ Although at that point I had not yet read [4].

${ }^{18}$ Although English speakers frequently use 'suppose' and 'assume' as synonyms, my own ideolect (perhaps corrupted by thinking about modal logic) prefers the indicative mood with 'assume that' and the subjunctive mood with 'suppose that'. Perhaps I am not unique in this regard. If so, one might refer to assumption under the marker $\mathbf{1}$ as supposition, since model-theoretically it bears on an accessible world, and reserve 'assume' for assumption under the marker $\mathbf{0 .}$ 


\subsection{Definitions}

Let $\Gamma \vdash_{I K} \chi\left[\Gamma \vdash_{I K}^{-} \chi\right]$ iff for some $C$ and $\mathcal{D}, C \Rightarrow \mathcal{D}: \chi\left[C \Rightarrow^{-} \mathcal{D}: \chi\right]$ and $\operatorname{ran}(C) \subseteq \Gamma$.

If $\Gamma$ is finite, I will follow the standard convention of listing the members of $\Gamma$ to the left of $\vdash_{I K}$ without the curly brackets when writing $\Gamma \vdash_{I K} \chi$; ditto for $\vdash_{I K}^{-}$.

If $C \Rightarrow \mathcal{D}: \chi$ or $C \Rightarrow^{-} \mathcal{D}: \chi$, let $A(\mathcal{D})=\operatorname{ran}(C) . A(\mathcal{D})$ is the set of $\mathrm{m}$-formulas assumed in $\mathcal{D}$.

\subsection{Remarks}

$\&, \vee, \supset$ and $\perp$ are what I call "level 0" logical constants, since their introduction and elimination rules involve no uses of any other logical constants. ${ }^{19}$ Were we to take $\neg$ as a primitive logical constant, it would be of "level 1", since its canonical elimination rule uses $\perp .{ }^{20}$ Our introduction and elimination rules for $\square$ and $\diamond$ show them to also be of level 1 , since they use $T$.

Our elimination rules for $\square$ and $\diamond$ invert (in the sense of Prawitz) the corresponding introduction rules - i.e. they are "harmonious" (in Michael Dummett's sense).

And they are sense-determining, in the following sense. Introduce a fresh monadic operator $\widehat{\square}$, and add introduction and elimination rules $\widehat{\square} I$ and $\widehat{\square} E$ by replacing $\square$ by $\widehat{\square}$ in the formulations of $\square I$ and $\square E$. In the resulting proof-theoretic system, and $\widehat{\square}$ are interchangeable, preserving deductions. Similarly for $\diamond$.

Our Natural Deduction system(s) can easily be transformed into Sequent Calculi, in which the right and left entry rules that correspond to our introduction and elimination rules for $\square$ and $\diamond$ are symmetric in the sense of Parisi. ${ }^{21}$

\section{Some Deductions and Remarks}

I will omit the subscript in $\vdash_{I K}$ until further notice.

\subsection{Lemmas}

The following rules are admissible for $\Rightarrow=\Rightarrow_{I K}$ or $\Rightarrow_{I K}^{-}$.

Transfer $_{m}$ If for $m \in \omega, m>0,\left\{v_{i}: \mathbf{0} \varphi_{i} \mid i \in(m)\right\} \Rightarrow \mathcal{D}_{0}: \mathbf{0} \theta$, for each $i \in$ (m) $C_{i} \Rightarrow \mathcal{D}_{i}: \mathbf{1} \varphi_{i}$, and $\left\{C_{i} \mid i \in(m)\right\}$ is coherent, then there is a $\mathcal{D}$ so that

${ }^{19}$ They are separated, in the sense of [11], p. 183.
${ }^{20}$ It has this form.
\[ \begin{array}{cc}\mathcal{D}_{1} \quad \mathcal{D}_{0} \\ \mathbf{0} \neg \varphi \quad \mathbf{0} \varphi \\ \perp\end{array} \]

${ }^{21}$ See [5], p. 44. 
$\bigcup_{i \in(m)} C_{i} \Rightarrow \mathcal{D}: \mathbf{1} \theta$. Here is the picture for $m=1$.

$$
\begin{aligned}
& \text { [ } \left.\nu: \mathbf{0} \varphi_{1}\right] \\
& \mathcal{D}_{0}
\end{aligned}
$$

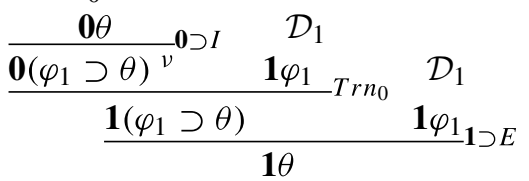

For $m>1$, iterate $\mathbf{0} \supset I m$-times, and then $\mathbf{1} \supset E m$-times. Let $\operatorname{Transfer}_{m}$ be this derived rule.

Transfer 1 lets us "complete" $1 \perp E_{\mathbf{0}}$ thus.

$1 \perp$ Elimination $_{1} \quad$ For $\theta \in F m l$, if $C \Rightarrow \mathcal{D}_{0}: \mathbf{1} \perp$ then $C \Rightarrow \mathcal{D}: \mathbf{1} \theta$ for $\mathcal{D}$ as pictured.

$$
\frac{\frac{[v: \mathbf{0} \perp]_{\mathbf{0}} \perp E}{\mathbf{0} \theta \quad \mathcal{D}_{0}}}{\mathbf{1} \perp^{v}} T r n_{1}
$$

Transfer $_{1}$ also gives us derived rules $\mathbf{1} \vee I$ and $1 \& E$ that, in an obvious sense, are 1 -versions of $\mathbf{0} \vee I$ and $0 \& E$. Here is one example; the remaining one is an execise for the reader.

$\mathbf{1} \vee$ Introduction $\quad$ for $i \in 2$, if $C \Rightarrow \mathcal{D}_{0}: \mathbf{1} \varphi_{i}$ then $C \Rightarrow \mathcal{D}: \mathbf{1}(\varphi \supset \psi)$ for this $\mathcal{D}$.

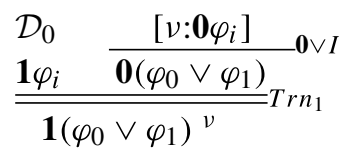

Transfer 2 gives us a $\mathbf{1}$-version of $0 \& I$ as a derived rule, as follows.

If for $i \in\{2\}, C_{i} \Rightarrow \mathcal{D}_{i}: \mathbf{1} \varphi_{i}$ and $\left\{C_{0}, C_{1}\right\}$ is coherent then $C \Rightarrow \mathcal{D}: \mathbf{1}\left(\varphi_{0} \& \varphi_{1}\right)$ for this $\mathcal{D}$.

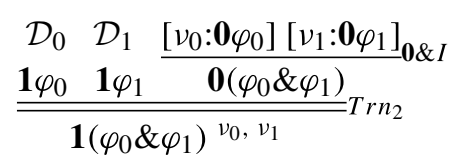

Remark We could have taken Transfer ${ }_{2}$ as primitive, and then obtained $\mathbf{1} \supset E$ as a derived rule. But our approach is more economical.

$\mathbf{1} \vee$ Elimination $_{1} \quad$ If $C_{2} \Rightarrow \mathcal{D}_{2}: \mathbf{1}\left(\varphi_{0} \vee \varphi_{1}\right)$, for both $i \in 2 C_{i}, v_{i}: \mathbf{1} \varphi_{i} \Rightarrow \mathcal{D}_{i}: \mathbf{1} \theta$, $\nu_{0}, \nu_{1} \in V a r$ are distinct, and $\left\{C_{0}, C_{1}, C_{2}\right\}$ is coherent, there is a deduction $\mathcal{D}$ so that $\bigcup_{i \in 3} C_{i} \Rightarrow \mathcal{D}: \mathbf{1} \theta$.

We will construct such a $\mathcal{D}$ as follows. Let $\sigma_{0}$ be $\left(\left(\varphi_{0} \supset \theta\right) \supset\left(\left(\varphi_{1} \supset \theta\right) \supset \theta\right)\right)$, and $\sigma_{1}$ be $\left(\left(\varphi_{0} \vee \varphi_{1}\right) \supset \sigma_{0}\right) . \sigma_{1}$ is an intuitionistic validity; so we may fix $\mathcal{D}^{\prime}$ to be the obvious deduction, constructed using $\mathbf{0} \vee E$ and $\mathbf{0} \supset I$, such that \{\}$\Rightarrow \mathcal{D}^{\prime}: \mathbf{0} \sigma_{1}$. Let $\mathcal{D}^{\prime \prime}$ be the following.

$$
\begin{array}{cc}
\mathcal{D}^{\prime} & \mathcal{D}_{2} \\
\mathbf{0} \sigma_{1} & \mathbf{1}\left(\varphi_{0} \vee \varphi_{1}\right) \\
\mathbf{1} \sigma_{1} & T r n_{0}
\end{array}
$$


For both $i \in 2$ let $\mathcal{D}_{i}^{\prime}$ be the following.

$$
\begin{aligned}
& {\left[\nu_{i}: \mathbf{1} \varphi_{i}\right]} \\
& \mathcal{D}_{i} \quad \mathcal{D}_{2} \\
& \frac{\mathbf{1} \theta \mathbf{1}\left(\varphi_{0} \vee \varphi_{1}\right)}{\mathbf{1}\left(\varphi_{i} \supset \theta\right)} \mathbf{1} \supset I
\end{aligned}
$$

Let $\mathcal{D}$ be the following.

$$
\begin{aligned}
& \mathcal{D}^{\prime \prime} \quad \mathcal{D}_{2}
\end{aligned}
$$

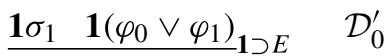

$$
\begin{aligned}
& {\underline{1} \sigma_{0} \quad \mathbf{1}\left(\varphi_{0} \supset \theta\right)}_{1} \supset E \quad \mathcal{D}_{1}^{\prime}
\end{aligned}
$$

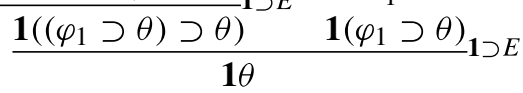

We may abbreviate $\mathcal{D}$ thus.

$$
\begin{aligned}
& {\left[v_{0}: \mathbf{1} \varphi_{0}\right]\left[v_{1}: \mathbf{1} \varphi_{1}\right]} \\
& \begin{array}{lll}
\mathcal{D}_{2} & \mathcal{D}_{0} & \mathcal{D}_{1}
\end{array} \\
& \frac{\mathbf{1}\left(\varphi_{0} \vee \varphi_{1}\right) \quad \mathbf{1} \theta \quad \mathbf{1} \theta}{\mathbf{1} \theta v^{v_{0}, v_{1}}} \mathbf{1} \vee E_{\mathbf{1}}
\end{aligned}
$$

The reader might amuse him/herself by computing $d p d(\mathcal{D})$.

Necessitation $\quad$ If \{\}$\Rightarrow \mathcal{D}_{0}: \mathbf{0} \varphi$, then \{\}$\Rightarrow \mathcal{D}: \mathbf{0} \square \varphi$ for the following $\mathcal{D}$.

$$
\begin{gathered}
\mathcal{D}_{0} \\
\frac{[\nu: \mathbf{1} \top] \quad \mathbf{0} \varphi}{\frac{\mathbf{1} \varphi}{\mathbf{0} \square \varphi^{\nu}}} \operatorname{Trn}_{0}{ }^{\nu}
\end{gathered}
$$

The barrier for the indicated use of $\square I$ is \{\} .

\subsection{Lemmas}

We will now consider some schematic deductions that, in effect, recover the axioms presented in [7].

(1) $\mathbf{0} \diamond \perp \vdash \mathbf{0} \perp$, witnessed by the following.

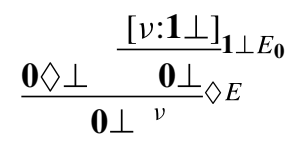

The barrier for the indicated use of $\diamond E$ is \{\} . 
(2) $\mathbf{0} \square(\varphi \supset \psi), \mathbf{0} \square \varphi \vdash \mathbf{0} \square \psi$, witnessed by the following. ${ }^{22}$

$$
\frac{\frac{\mu_{0}: \mathbf{0} \square(\varphi \supset \psi)[v: \mathbf{1} \top]}{\mathbf{1}(\varphi \supset \psi)} \square E \frac{\mu_{1}: \mathbf{0} \square \varphi[\nu: \mathbf{1} \top]}{\mathbf{1} \varphi_{1}} \square E}{\frac{\mathbf{1} \psi}{\mathbf{0} \square \psi^{v}} \square I}
$$

The barrier for the indicated use of $\square I$ is \{\} .

(3) $\mathbf{0} \square(\varphi \supset \psi), \mathbf{0} \diamond \varphi \vdash \mathbf{0} \diamond \psi$, witnessed by the following.

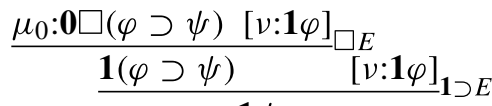

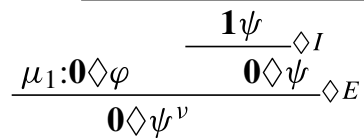

The barrier for the indicated use of $\diamond E$ is \{\} .

(4) $\mathbf{0}(\diamond \varphi \supset \square \psi) \vdash \mathbf{0} \square(\varphi \supset \psi)$, witnessed by the following

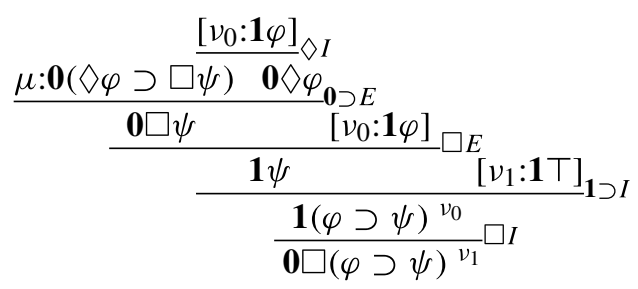

The discharging of $v: \mathbf{1} \top$ at $[0,0]$ is vacuous. The barrier for the indicated use of $\square I$ is \{\} .

(5) $\mathbf{0} \diamond(\varphi \vee \psi) \vdash \mathbf{0}(\diamond \varphi \vee \diamond \psi)$, witnessed by the following.

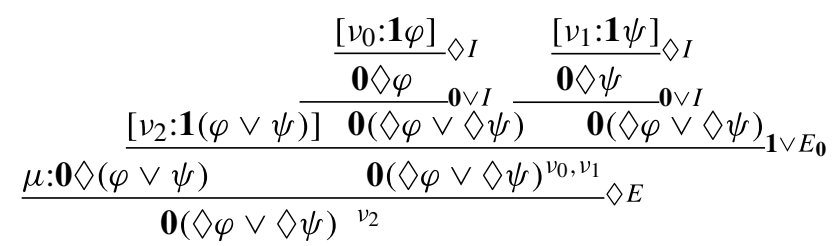

The barrier for the indicated use of $\diamond E$ is \{\} .

\subsection{Observation}

For each axiom $\psi$ of Plotkin and Sterling, $\vdash_{I K} \mathbf{0} \psi$. Proof: apply $\mathbf{0} \supset I$ as needed to (1)-(5) above.

\subsection{Observations}

A few other examples may be helpful.

\footnotetext{
${ }^{22}$ This is, of course, the $\mathbf{0}$-version of the K-rule.
} 
(1) $1 \varphi \vdash_{I K} \mathbf{1} \top$, witnessed by the following.

$$
\frac{[v: \mathbf{1} \varphi] \quad \overline{\mathbf{0} \top}}{\mathbf{1} \top} \operatorname{Trn}_{0}
$$

(2) $\mathbf{0} \square \varphi, \mathbf{0} \diamond \psi \vdash_{I K} \mathbf{0} \diamond(\varphi \& \psi)$, witnessed by the following.

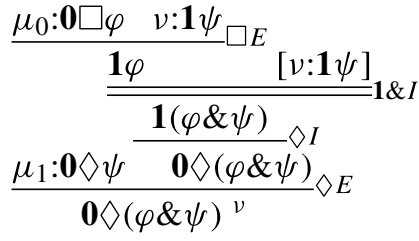

The barrier for the indicated use of $\diamond E$ is \{\} .

\subsection{Exercises}

The reader might like to construct deductions in IK to witness the following: $\vdash_{I K}$ $\mathbf{0} \square \top ; \mathbf{0} \neg \diamond \top \vdash_{I K} \mathbf{0} \square \perp ; \mathbf{0} \square \perp, \mathbf{0} \diamond \top \vdash_{I K} \mathbf{0} \perp$.

\subsection{Observation}

Consider a set $\Gamma$ of formulas and a formula $\varphi$ containing no occurrences of $\square$ or $\diamond$, and an intuitionistic ND deduction $\mathcal{D}$ of $\varphi$ from $\Gamma$ using the familiar "no step" rules. Form $\Gamma^{\prime}, \varphi^{\prime}$ and $\mathcal{D}^{\prime}$ from $\Gamma, \varphi$ and $\mathcal{D}$ respectively by uniformly substituting formulas (which may contain occurrences of $\square$ or $\diamond$ ) for members of $\mathcal{S}$. Form $\mathcal{D}^{\prime \prime}$ from $\mathcal{D}^{\prime}$ by prefixing the formula-label for each string in $\operatorname{dom}\left(\mathcal{D}^{\prime}\right)$ by $\mathbf{0}$. Then $\mathcal{D}^{\prime \prime}$ witnesses that $\mathbf{0} \Gamma^{\prime} \vdash_{I K} \mathbf{0} \varphi^{\prime}$.

\section{Substitution and Amputation in Deductions}

\subsection{Definitions}

Consider $C, C^{\prime}, \mathcal{D}, \mathcal{D}^{\prime}, \chi, \chi^{\prime}$ and $\nu$ so that $C, \nu: \chi^{\prime} \Rightarrow \mathcal{D}: \chi, C^{\prime} \Rightarrow \mathcal{D}^{\prime}: \chi^{\prime}$, and $\left\{C, C^{\prime}\right\}$ is coherent.

\subsection{Definitions}

Informally, $\left[v:=\mathcal{D}^{\prime}\right] \mathcal{D}$ is the labeled tree formed by splicing a copy of $\mathcal{D}^{\prime}$ into $\mathcal{D}$ at every leaf in $\operatorname{dpd}(\mathcal{D})$ with label $v: \chi^{\prime}$. As the notation indicates, $\left[v:=\mathcal{D}^{\prime}\right]$ is a substitution function. Formally, we define $\left[v:=\mathcal{D}^{\prime}\right]$ by induction on the stages of the inductive definition of $\Rightarrow$.

Base clause: if $\mathcal{D}=\{\langle[], \mu: \chi\rangle\}$ then

$$
\left[v:=\mathcal{D}^{\prime}\right] \mathcal{D}= \begin{cases}\mathcal{D}^{\prime} \text { if } v: \chi^{\prime}=\mu: \chi \\ \mathcal{D} \text { otherwise }\end{cases}
$$


For the induction step, the definition is straightforward. (Reminder: discharging, as indicated by variable-superscripts at root, in effect binds occurrences of the variable(s) at leaves in $\operatorname{dpd}(\mathcal{D})$ with label(s) tagged by that variable, and this blocks substitution.)

\subsection{Remarks}

In some cases, $\left[v:=\mathcal{D}^{\prime}\right] \mathcal{D}$ will be "bad" (i.e. not what we want), by virtue of failure of substitutability: a tag in a label of some undischarged leaf of $\mathcal{D}^{\prime}$ may get bound after substitution by some discharging step within $\mathcal{D} .^{23}$ But "bound" (i.e. discharging) tags in $\mathcal{D}$ can be "relettered", i.e. replaced by distinct "fresh" tags (variables not occurring in $\mathcal{D}$ ), to form a new but slightly different deduction. So we can define a revised substitution function $\left[\mathcal{D}^{\prime} / \nu\right]$ as follows.

\subsection{Definition}

Fix an $\omega$-ordering of $\operatorname{Var}$. Given a deduction $\mathcal{D}$, let $\mu_{1}, \ldots, \mu_{m}$ be the variables (listed without repetitions) that tag the labels of members of $d p d\left(\mathcal{D}^{\prime}\right)$ and also that occur discharged (i.e. bound) in $\mathcal{D}$. Fix $\mu_{1}^{\prime}, \ldots, \mu_{m}^{\prime} \in \operatorname{Var}$ that don't occur in $\mathcal{D}$ and are not in $\left\{\mu_{i \in(m)}\right\}$; do this in some unique way, e.g. $\mu_{1}^{\prime}$ is the first such variable in the ordering of $V a r$, , then $\mu_{2}^{\prime}$ is the next, etc. Form $\mathcal{D}^{*}$ from $\mathcal{D}$ by replacing occurrences of $\mu_{i}$ by $\mu_{i}^{\prime}$, for $i \in(m)$, say from left to right and high to low (this to make $\mathcal{D}^{*}$ unique). Note: $C \Rightarrow \mathcal{D}^{*}: \chi$. We have made sure that applying $\left[v:=\mathcal{D}^{\prime}\right]$ to $\mathcal{D}^{*}$ involves no failure of substitutibility. Let $\left[\mathcal{D}^{\prime} / \nu\right] \mathcal{D}=\left[\nu:=\mathcal{D}^{\prime}\right] \mathcal{D}^{*}$.

\subsection{Observation}

In the above situation, $C \cup C^{\prime} \Rightarrow\left[\mathcal{D}^{\prime} / \nu\right] \mathcal{D}: \chi$. Proof is by induction on the height of $\mathcal{D}$.

\subsection{Definitions}

Let $\vdash$ be a consequence relation (on $M F m l$ ) iff $\vdash \subseteq \operatorname{Power}(M F m l) \times M F m l$ and $\vdash$ satisfies these conditions: (i) (reiteration) for any $\chi \in M F m l\{\chi\} \vdash \chi$; (ii) (cut) for any $\Gamma_{0}, \Gamma_{1} \subseteq M F m l$ and $\chi \in M F m l$, if $\Gamma_{0} \vdash \gamma$ for every $\gamma \in \Gamma_{1}$ and $\Gamma_{0} \cup \Gamma_{1} \vdash \chi$ then $\Gamma_{0} \vdash \chi$.

Consider a consequence relation $\vdash . \vdash$ is monotonic iff for any $\Gamma_{0}, \Gamma_{1} \subseteq M F m l$ and $\chi \in M F m l$, if $\Gamma_{0} \vdash \chi$ and $\Gamma_{0} \subseteq \Gamma_{1}$ then $\Gamma_{1} \vdash \chi . \vdash$ is finitary iff for any $\Gamma \subseteq M F m l$ and $\chi \in M F m l$, if $\Gamma \vdash \chi$ then for some finite $\Gamma_{1} \subseteq \Gamma_{0}, \Gamma_{1} \vdash \chi$.

For $\varphi \in F m l$, distinct $\alpha_{1}, \ldots, \alpha_{n} \in S$, and $\psi_{1}, \ldots, \psi_{n} \in F m l$, let $[\vec{v} ; \vec{\psi}] \varphi$ be the result of simultaneously substituting $\psi_{i}$ for all occurrences of $\alpha_{i}$ in $\varphi$, for

\footnotetext{
${ }^{23}$ This is analogous to failure of substitutibility in a first-order language when a variable occurring in a term that is substituted into a formula gets bound by a quantifier-prefix occurring in that formula. One difference: $\left[v:=\mathcal{D}^{\prime}\right] \mathcal{D}$ could even fail to be a deduction relative to $C_{0} \cup C_{1}$ (because of a mismatch of types, i.e. of formulas paired with bound variables).
} 
$i \in(n)$. Similarly for $\chi \in F m l$ and $[\vec{v} ; \vec{\psi}] \chi$. For $\Gamma \subseteq M F m l,[\vec{v} ; \vec{\psi}] \Gamma=$ $\{[\vec{v} ; \vec{\psi}] \gamma \mid \gamma \in \Gamma\} . \vdash$ is structural iff for any $\Gamma \subseteq M F m l, \chi \in M F m l$, and $\vec{v}$ and $\vec{\psi}$ as above, if $\Gamma \vdash \chi$ then $[\vec{v} ; \vec{\psi}] \Gamma \vdash[\vec{v} ; \vec{\psi}] \chi \cdot{ }^{24}$

\subsection{Corollary}

$\vdash_{I K}$ is a finitary, monotonic structural consequence relation on $M F m l$. Proof: the previous observation insures satisfaction of the cut condition.

\subsection{Remark}

The following questions might have occurred to an attentive reader. In presenting $\square I$, why not require that $A\left(\mathcal{D}_{0}\right)-\{v: \mathbf{1} \top\} \subseteq \mathbf{0} F m l$ ? And in presenting $\diamond E$, why not require that $\operatorname{ran}\left(C_{0}-\{v: \mathbf{1} \varphi\}\right) \subseteq \mathbf{0} F m l$ ? Both of these changes would have made our formulation of these rules simpler than imposing the barrier conditions. The answer: these changes would block 5.5, and thus block 5.7.

\subsection{Definitions}

Assume that $C, \nu_{1}: \chi_{1}, \ldots, v_{n}: \chi_{n} \Rightarrow \mathcal{D}: \chi$, for each $i \in(n) C_{i}^{\prime} \Rightarrow \mathcal{D}_{i}^{\prime}: \chi_{i}^{\prime}$, and $\{C\} \cup$ $\bigcup_{i \in(n)} C_{i}^{\prime}$ is coherent. In this case, we define $\left[v_{1}, \ldots, v_{n}:=\mathcal{D}_{1}^{\prime}, \ldots, \mathcal{D}_{n}^{\prime}\right] \mathcal{D}$, the result of simultaneously substituting each $\mathcal{D}_{i}^{\prime}$ for $v_{i}, i \in(n)$, into $\mathcal{D}$, by generalizing in the obvious way the case for $n=1$. We define $\left[\mathcal{D}_{1}^{\prime}, \ldots, \mathcal{D}_{n}^{\prime} / \nu_{1}, \ldots, v_{n}\right] \mathcal{D}$ similarly.

\subsection{Definitions}

Consider $C, \mathcal{D}$ and $\chi$ so that $C \Rightarrow \mathcal{D}: \chi$, and $s_{1}, \ldots, s_{m} \in \operatorname{dom}(\mathcal{D})$, listed without repetitions. Assume that for each $i \in(m) s_{i}$ is open in $\mathcal{D}$ and $\left\{s_{i \in(m)}\right\}$ is an anti-chain in $\operatorname{dom}(\mathcal{D})$. For $i \in(m)$, let $\chi_{i}$ be the $\mathrm{m}$-formula label of $s_{i}$ in $\mathcal{D}$. Consider distinct $v_{1}, \ldots, v_{m} \in \operatorname{Var}$, none occurring in $\mathcal{D}$. Let $\mathcal{D}^{\$}$, the result of surgery on $\mathcal{D}$ at $s_{1}, \ldots, s_{m}$ using $v_{1}, \ldots, v_{m}$, be as follows: $\operatorname{dom}\left(\mathcal{D}^{\$}\right)=\operatorname{dom}(\mathcal{D})-\left\{s \mid\right.$ for some $\left.i \in(m) s_{i} \preceq s\right\}$; $\mathcal{D}^{\$}(s)=\mathcal{D}(s)$ for each $s \in \operatorname{dom}\left(\mathcal{D}^{\$}\right)$ such that $s \notin\left\{s_{i \in(m)}\right\}$; for each $i \in(m)$ let $\mathcal{D}^{\$}\left(s_{i}\right)=\mu_{i}: \chi_{i}$.

For $i \in(m)$, let $\mathcal{D}_{i}$ be the subdeduction of $\mathcal{D}$ with root at $s_{i}$, i.e. $\mathcal{D}_{i}=\mathcal{D}_{s_{i}}$. In the above surgery on $\mathcal{D}$, each $\mathcal{D}_{i}$ has been amputated at $s_{i}$, with $v_{i}$ as a bandage on $s_{i}$.

\subsection{Observation}

For some $C_{i} \subseteq C, C_{i} \Rightarrow \mathcal{D}_{i}: \chi_{i}$. Note: this required that $s_{i}$ be open in $\mathcal{D}$ (i.e. no leaf of $\mathcal{D}$ above $s_{i}$ was discharged in $\mathcal{D}$ ).

\footnotetext{
${ }^{24}$ See $[11]$
} 


\subsection{Observation}

Let $C^{\$}=\left\{\mu: \xi \mid\right.$ for some $s \in d p d(\mathcal{D})$, no $i \in(m)$ has $s_{i} \preceq s$ and $\left.\mathcal{D}(s)=\mu: \xi\right\}$. Then $C^{\$} \cup\left\{v_{i}: \chi_{i} \mid i \in(m)\right\} \Rightarrow \mathcal{D}^{\$}: \chi$.

\subsection{Observation}

This surgery can be reversed:

$$
[\vec{v}:=\overrightarrow{\mathcal{D}}] \mathcal{D}^{\$}=[\overrightarrow{\mathcal{D}} / \overrightarrow{\mathcal{v}}] \mathcal{D}^{\$}=\mathcal{D}
$$

\subsection{Theorem}

For $\Sigma \cup\{\theta\} \subseteq F m l, \mathbf{0} \Sigma \vdash_{I K} \mathbf{0} \theta$ iff $\mathbf{0} \Sigma \vdash_{I K}^{-} \mathbf{0} \theta$.

Proof. Right to left is trivial. Assume the left-side. Fix $C$ and $\mathcal{D}$ so that $\operatorname{ran}(C) \subseteq$ $\mathbf{0} \Sigma$ and $C \Rightarrow_{I K} \mathcal{D}: \mathbf{0} \theta$. If there are no uses of $\nabla E_{+}$in $\mathcal{D}$, we have the right-side for free. Assume that $s_{0} \in \operatorname{dom}(\mathcal{D})$ was entered using $\diamond E^{+}$; so $\mathcal{D}\left(s_{0}\right)=1 \top$ and $\mathcal{D}\left(\langle 0\rangle s_{0}\right)=\mathbf{0} \diamond \top$. Fix $v \in \operatorname{Var}$ not occurring in $\mathcal{D}$. Let $\mathcal{D}^{\$}$ be the result of surgery on $\mathcal{D}$ at $s$ using $v$ as the bandage. Let $\mathcal{D}_{s_{0}}$ be the sub-deduction so amputated, and let $\mathcal{D}_{\langle 0\rangle} \wedge_{0}$ be its immediate proper subdeduction. So $s \in \operatorname{dom}\left(\mathcal{D}_{s_{0}}\right)$ iff $s^{\wedge} s_{0} \in \operatorname{dom}(\mathcal{D})$, and $s \in \operatorname{dom}\left(\mathcal{D}_{\langle 0\rangle} s_{0}\right)$ iff $s^{\wedge}\langle 0\rangle s_{0} \in \operatorname{dom}(\mathcal{D})$. Also, $\left.\mathcal{D}_{\langle 0\rangle} \wedge s\right)=\mathcal{D}\left(s^{\wedge}\langle 0\rangle s_{0}\right)$. So for some $C_{0}$ and $C^{\$}, C=C_{0} \cup C^{\$}, C_{0} \Rightarrow \mathcal{D}_{\langle 0\rangle} \wedge_{0}: \mathbf{0} \diamond \top, C^{\$}, v: \mathbf{1} \top \Rightarrow \mathcal{D}^{\$}: \mathbf{0} \theta$, and $\operatorname{ran}\left(C^{\$}\right) \subseteq \mathbf{0} F m l$. Construct $\mathcal{D}_{1}$ using $\diamond E_{0}$ as pictured.

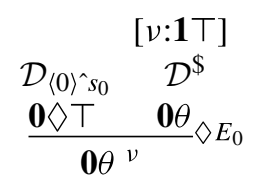

So $C \Rightarrow_{I K} \mathcal{D}^{\prime}: \mathbf{0} \theta$, and $\mathcal{D}^{\prime}$ contains one fewer use of $\diamond E^{+}$than did $\mathcal{D}$. Iterate this procedure until all uses of $\diamond E^{+}$have been eliminated.

\section{Soundness of IK}

For $\Gamma \subseteq M F m l$ let $\boldsymbol{m}^{-1} \Gamma=\{\varphi \mid \boldsymbol{m} \varphi \in \Gamma\}$

\subsection{Soundness}

Theorem for $I K$. If $\Gamma \vdash \chi$ then $\langle\Gamma, \chi\rangle$ is IK-valid.

Consider any IK-model $\mathcal{M}$ with $F^{\mathcal{M}}=\langle W, R$, $\rangle$. It suffices to prove the following: for any $C, \mathcal{D}$ and $\chi$, if $C \Rightarrow \mathcal{D}: \chi$ then $\langle A(\mathcal{D}), \chi\rangle$ is $\mathcal{M}$-valid.

We do this by induction on the stages of the inductive definition of $\Rightarrow$, equivalently on the height of $\mathcal{D}$, call it $h t(\mathcal{D})$. Restate our goal thus: for every $n \in \omega$, every $C, \mathcal{D}$ and $\chi$, if $C \Rightarrow \mathcal{D}: \chi$ and $h t(\mathcal{D}) \leq n$, then for every $u \in W^{\mathcal{M}},(!)\langle A(\mathcal{D}), \chi\rangle$ is $\mathcal{M}$-valid at $u$. 
The base step is trivial. Given $n \in \omega$, assume the obvious Induction Hypothesis regarding the $n$th stage the inductive definition of $\Rightarrow$. Consider $C, \mathcal{D}, \chi$ and $u \in W$ so that $C \Rightarrow \mathcal{D}: \chi$ and $h t(\mathcal{D}) \leq n+1$. We need a soundness lemma for each rule by which [ ] might have been entered into $\mathcal{D}$.

In what follows, let (A) be this: $u$ is a dead-end and $\mathcal{M}, u \Vdash A(\mathcal{D})$. And let (B) be this: given $v, \mathcal{M}, u, v \Vdash A(\mathcal{D})$.

Except for $\mathbf{0} \supset I$, the soundness lemmas for the introduction and elimination rules for the Boolean constants governed by $\mathbf{0}$ are relatively straightforward; so I will skip them.

Assume that [ ] was entered into $\mathcal{D}$ by $\mathbf{0} \supset I$, as pictured in 3.8. By the $\mathrm{IH}$, (*) $\left\langle A\left(\mathcal{D}_{0}\right), \mathbf{0} \psi\right\rangle$ is $\mathcal{M}$-valid at $u$. Assume $(\mathrm{A})$. So $A(\mathcal{D}) \subseteq \mathbf{0} F m l$ and $\mathcal{M}, u \models$ $\mathbf{0}^{-1} A(\mathcal{D})$. Consider a $u^{\prime} \sqsupseteq u$ such that $\mathcal{M}, u^{\prime}=\varphi$. By the Persistence Lemma, $\mathcal{M}, u^{\prime} \models \mathbf{0}^{-1} A(\mathcal{D})$; so $\mathcal{M}, u^{\prime}=\mathbf{0}^{-1} A\left(\mathcal{D}_{0}\right)$. So $\mathcal{M}, u^{\prime} \Vdash A\left(\mathcal{D}_{0}\right)$. By (*) $\mathcal{M}, u^{\prime} \Vdash$ $\mathbf{0} \psi$; so $\mathcal{M}, u^{\prime} \models \psi$. So $\mathcal{M}, u \models(\varphi \supset \psi)$. Assume (B). Consider a $u^{\prime} \sqsupseteq u$ such that $\mathcal{M}, u^{\prime} \models \varphi$. Again, by the Persistence Lemma, $\mathcal{M}, u^{\prime}=\mathbf{0}^{-1} A(\mathcal{D})$. But what about $1^{-1} A(\mathcal{D})$ ? By the right-completeness of $F^{\mathcal{M}}$ we can fix a $v^{\prime} \sqsupseteq v$ so that $u^{\prime} R v^{\prime}$. Since $\mathcal{M}, v \models \mathbf{1}^{-1} A(\mathcal{D})$, by the Persistence Lemma $\mathcal{M}, v^{\prime}=\mathbf{1}^{-1} A(\mathcal{D})$; so $\mathcal{M}, u^{\prime}, v^{\prime} \Vdash A(\mathcal{D})$. Since $\mathcal{M}, u^{\prime}, v^{\prime} \Vdash \mathbf{0} \varphi, \mathcal{M}, u^{\prime}, v^{\prime} \Vdash A\left(\mathcal{D}_{0}\right)$. By $\left(^{*}\right) \mathcal{M}, u^{\prime}, v^{\prime} \Vdash$ $\mathbf{0} \psi$; so $\mathcal{M}, u^{\prime} \models \psi$. So $\mathcal{M}, u \models(\varphi \supset \psi)$. (!) follows.

Assume that [ ] was entered into $\mathcal{D}$ by $\mathbf{1} \perp E_{\mathbf{0}}$, as pictured in 3.8. By the $\mathrm{IH},\left(^{*}\right)$ $\left\langle A\left(\mathcal{D}_{0}\right), \mathbf{1} \perp\right\rangle$ is $\mathcal{M}$-valid at $u$. Assume $(\mathrm{A})$. By (*) $\mathcal{M}, u \Vdash \mathbf{1} \perp$; so $\mathbf{1} \perp \in \mathbf{0} F m l$, a contradiction; so $\mathcal{M}, u \nVdash A(\mathcal{D})$. (V1) follows vacuously. Assume (B). By (*) $\mathcal{M}, u, v \Vdash \mathbf{1} \perp$; so $\mathcal{M}, v \models \perp$, a contradiction; (V2) vacuously follows, yielding (!).

Assume that [ ] was entered into $\mathcal{D}$ by $1 \vee E_{\mathbf{0}}$, as pictured in 3.8. By the IH, (*) $\left\langle A\left(\mathcal{D}_{2}\right), \mathbf{1}\left(\varphi_{0} \vee \varphi_{1}\right)\right\rangle$ and $(* *)$ for both $i \in 2\left\langle A\left(\mathcal{D}_{i}\right), \mathbf{0} \theta\right\rangle$ are $\mathcal{M}$-valid at $u$. Assume (A). Since $\mathcal{M}, u \Vdash A\left(\mathcal{D}_{2}\right)$, by $(*) \mathcal{M}, u \Vdash \mathbf{1}\left(\varphi_{0} \vee \varphi_{1}\right)$; so $\mathbf{1}\left(\varphi_{0} \vee \varphi_{1}\right) \in \mathbf{0} F m l$, a contradiction; so $\mathcal{M}, u \nVdash A(\mathcal{D})$. (V1) follows vacuously. Assume (B). By (*), $\mathcal{M}, u, v \Vdash \mathbf{1}\left(\varphi_{0} \vee \varphi_{1}\right)$; so $\mathcal{M}, v \models\left(\varphi_{0} \vee \varphi_{1}\right)$. Fix $i \in 2$ so that $\mathcal{M}, v \models \varphi_{i}$. So $\mathcal{M}, u, v \Vdash \mathbf{1} \varphi_{i}$; so $\mathcal{M}, u, v \Vdash A\left(\mathcal{D}_{i}\right)$. By (**), $\mathcal{M}, u, v \Vdash \mathbf{0} \theta$, yielding (V2), and thus (!).

Assume that [ ] was entered into $\mathcal{D}$ by $1 \supset E$, as pictured in 3.8. By the $\mathrm{IH}(*)$ $\left\langle A\left(\mathcal{D}_{0}\right), \mathbf{1} \psi\right\rangle$ and $(* *)\left\langle A\left(\mathcal{D}_{1}\right), \mathbf{1}(\psi \supset \varphi)\right\rangle$ are $\mathcal{M}$-valid at $u$. Assume (A). By (*) $\mathcal{M}, u \Vdash \mathbf{1} \psi$, a contradiction. (V1) follows vacuously. Assume (B). By (*), $\mathcal{M}, u, v \Vdash$ $\mathbf{1} \psi$; by (ii) $\mathcal{M}, u, v \Vdash \mathbf{1}(\psi \supset \varphi)$; so $\mathcal{M}, v \models\{\psi,(\psi \supset \varphi)\}$; so $\mathcal{M}, v \models \varphi$; so $\mathcal{M}, u, v \Vdash \mathbf{1} \varphi$. (V2) follows, yielding (!).

Assume that [ ] was entered into $\mathcal{D}$ by $1 \supset I$, as pictured in 3.8. By the $\mathrm{IH}(*)$ $\left\langle A\left(\mathcal{D}_{0}\right), \mathbf{1} \psi\right\rangle$ and $\left({ }^{* *}\right)\left\langle A\left(\mathcal{D}_{1}\right), \mathbf{1} \theta\right\rangle$ are $\mathcal{M}$-valid at $u$. Assume (A). By (**), $\mathcal{M}, u \Vdash$ $1 \theta$, a contradiction. (V1) follows vacuously. ${ }^{25}$ Assume (B). Consider a $v^{\prime}$ so that $v \sqsubseteq v^{\prime}$; assume that $\mathcal{M}, v^{\prime} \models \varphi$. Since $\langle W, R$, $\rangle$ is left-complete, we may fix a $u^{\prime}$ so that $u \sqsubseteq u^{\prime} R v^{\prime}$. Since $\mathcal{M}, u=\mathbf{0}^{-1} A\left(\mathcal{D}_{0}\right)$ and $\mathcal{M}, v \models \mathbf{1}^{-1} A\left(\mathcal{D}_{0}\right)-\{\varphi\}$, by the Persistence Lemma $\mathcal{M}, u^{\prime} \models \mathbf{0}^{-1} A\left(\mathcal{D}_{0}\right)$ and $\mathcal{M}, v^{\prime}=\mathbf{1}^{-1} A\left(\mathcal{D}_{0}\right)-\{\varphi\}$. Thus $\mathcal{M}, u^{\prime}, v^{\prime} \Vdash A\left(\mathcal{D}_{0}\right)$. By (*) $\mathcal{M}, u^{\prime}, v^{\prime} \Vdash \mathbf{1} \psi$; so $\mathcal{M}, v^{\prime}=\psi$; so $\mathcal{M}, v \models(\varphi \supset \psi)$. So $\mathcal{M}, u, v \Vdash \mathbf{1}(\varphi \supset \psi)$. (V2) follows, yielding (!).

\footnotetext{
${ }^{25}$ In effect, the minor premise $\mathbf{1} \theta$ says "No dead-end!".
} 
Assume that [ ] was entered into $\mathcal{D}$ by $\operatorname{Tr}_{0}$, as pictured in 3.8. Recall: \{\}$\Rightarrow$ $\mathcal{D}_{0}: \mathbf{0} \varphi$. By the $\mathrm{IH}(*)\left\langle A\left(\mathcal{D}_{1}\right), \mathbf{1} \theta\right\rangle$ is $\mathcal{M}$-valid at $u$. Assume (A). By (*) $\mathcal{M}, u \Vdash \mathbf{1} \theta$, a contradiction. (V1) vacuously follows. Assume (B). By the IH, (**) $\langle\{\}, \mathbf{0} \varphi\rangle \mathcal{M}$-valid at $v$. (Notice the switch from $u$ to $v$.) If $v$ is a dead-end, by (V1) for (**) $\mathcal{M}, v \Vdash \mathbf{0} \varphi$; so $\mathcal{M}, v=\varphi$. Assume that $v$ is not a dead-end; fix $w$ so that $v R w$. By (V2) for (**) $\mathcal{M}, v, w \Vdash \mathbf{0} \varphi$; so $\mathcal{M}, v \models \varphi$. So $\mathcal{M}, u, v \Vdash \mathbf{1} \varphi$. (V2) follows, yielding (!).

Assume that [ ] was entered into $\mathcal{D}$ by $\diamond I$, as pictured in 3.8. So $A(\mathcal{D})=A\left(\mathcal{D}_{0}\right)$. By the $\mathrm{IH}\left(^{*}\right)\left\langle A\left(\mathcal{D}_{0}\right), \mathbf{1} \varphi\right\rangle$ is $\mathcal{M}$-valid at $u$. Assume (A). By (*) $\mathcal{M}, u \Vdash \mathbf{1} \varphi$; so $\mathbf{1} \varphi \in$ $\mathbf{0} F m l$, for a contradiction. (V1) follows vacuously. Assume (B). By (*) $\mathcal{M}, u, v \Vdash$ $\mathbf{1} \varphi$; so $\mathcal{M}, v \models \varphi$; since $u R v, \mathcal{M}, u \models \diamond \varphi$; so $\mathcal{M}, u, v \Vdash \mathbf{0} \nabla \varphi$, proving (V2). (!) follows.

Assume that [ ] was entered into $\mathcal{D}$ by $\diamond E$, as pictured in 3.8; recall: $\mathcal{D}_{0}$ has a barrier with exception for $v: \mathbf{1} \varphi$. By the $\mathrm{IH},\left(^{*}\right)\left\langle A\left(\mathcal{D}_{1}\right), \mathbf{0} \nabla \varphi\right\rangle$ is $\mathcal{M}$-valid at $u$. Assume (A). By (*) $\mathcal{M}, u \Vdash \mathbf{0} \diamond \varphi$; so $\mathcal{M}, u \models \diamond \varphi$, a contradiction. (V1) vacuously follows. Assume (B). By (*) $\mathcal{M}, u, v \Vdash \mathbf{0} \diamond \varphi$; so $\mathcal{M}, u \models \Delta \varphi$. Fix $w$ so that $u R w$ and $\mathcal{M}, w \models \varphi$. So $\mathcal{M}, u, w \Vdash \mathbf{1} \varphi$. Fix $\left\{s_{i \in(m)}\right\}$ to be a barrier in $\mathcal{D}_{0}$ with exception for $\nu: 1 \varphi$. Fix distinct $v_{1}, \ldots, v_{m} \in \operatorname{Var}$, none occurring in $\mathcal{D}$. Let $\mathcal{D}_{0}^{\$}=$ the result of surgery on $\mathcal{D}_{0}$ at $s_{1}, \ldots, s_{m}$ using $v_{1}, \ldots, v_{m}$. For each $i \in(m)$ fix $\theta_{i}$ so that $\mathbf{0} \theta_{i}$ is the m-formula label of $s_{i}$ in $\mathcal{D}_{0}$, and let $\mathcal{D}_{i}^{\prime}$ be the subdeduction of $\mathcal{D}_{0}$ with root at $s_{i}$. Consider any $i \in(m)$. Since $s_{i}$ is open in $\mathcal{D}_{0}, A\left(\mathcal{D}_{i}^{\prime}\right) \subseteq A\left(\mathcal{D}_{0}\right)$; so $\mathcal{M}, u, v \Vdash A\left(\mathcal{D}_{i}^{\prime}\right)$. Since $h t\left(\mathcal{D}_{i}^{\prime}\right) \leq h t\left(\mathcal{D}_{0}\right)$, by the $\mathrm{IH},\left\langle A\left(\mathcal{D}_{i}^{\prime}\right), \mathbf{0} \theta_{i}\right\rangle$ is $\mathcal{M}$-valid at $u$. So $\mathcal{M}, u, v \Vdash \mathbf{0} \theta_{i}$. By choice of $\left\{s_{i \in(m)}\right\}$ and $\mathcal{D}_{0}^{\$}$,

$$
A\left(\mathcal{D}_{0}^{\$}\right) \subseteq\left(A\left(\mathcal{D}_{0}\right) \cap \mathbf{0} F m l\right) \cup\left\{\mathbf{0} \theta_{i \in(m)}\right\} \cup\{\mathbf{1} \varphi\} .
$$

So $\mathcal{M}, u \models \mathbf{0}^{-1} A\left(\mathcal{D}_{0}^{\$}\right)$. So $\mathcal{M}, u, w \Vdash A\left(\mathcal{D}_{0}^{\$}\right)$. Since $h t\left(\mathcal{D}_{0}^{\$}\right) \leq h t\left(\mathcal{D}_{0}\right) \leq n$, the IH insures that $\left\langle A\left(\mathcal{D}_{0}^{\$}\right), \mathbf{0} \psi\right\rangle$ is $\mathcal{M}$-valid at $u$. So $\mathcal{M}, u, w \Vdash \mathbf{0} \psi$; so $\mathcal{M}, u \models \psi$; so $\mathcal{M}, u, v \Vdash \mathbf{0} \psi$. (V2) follows, yielding (!).

Assume that [ ] was entered into $\mathcal{D}$ by $\square I$, as pictured in 3.8; recall: $\mathcal{D}_{0}$ has a barrier with exception for $v: \mathbf{1} T$. By the $\mathrm{IH}(*)\left\langle A\left(\mathcal{D}_{0}\right), \mathbf{1} \varphi\right\rangle$ is $\mathcal{M}$-valid at $u$. Assume (A). Given $v$, assume that $u R^{+} v{ }^{26}$ fix a $u^{\prime}$ so that $u \sqsubseteq u^{\prime} R v$. Since $\mathcal{M}, u \models \mathbf{0}^{-1} A(\mathcal{D})$, by the Persistence Lemma $\mathcal{M}, u^{\prime} \models \mathbf{0}^{-1} A(\mathcal{D})$. So $\mathcal{M}, u^{\prime} \Vdash A(\mathcal{D})$. Since $\mathcal{M}, v \models \top$, $\mathcal{M}, u^{\prime}, v \Vdash \mathbf{1} \top$; so $\mathcal{M}, u^{\prime}, v \Vdash A\left(\mathcal{D}_{0}\right)$. By the $\mathrm{IH},\left\langle A\left(\mathcal{D}_{0}\right), \mathbf{1} \varphi\right\rangle$ is $\mathcal{M}$-valid at $u^{\prime}$. So $\mathcal{M}, u^{\prime}, v \Vdash 1 \varphi$; so $\mathcal{M}, v \models \varphi$. Thus $\mathcal{M}, u \models \square \varphi$. (V1) follows. Assume (B). So $\mathcal{M}, u, v \Vdash A\left(\mathcal{D}_{0}\right)$. Fix $\left\{s_{i}\right\}_{i \in(m)}$ to be a barrier in $\mathcal{D}_{0}$ with exception for $v: \mathbf{1} \top$. Fix distinct $v_{1}, \ldots, v_{m} \in \operatorname{Var}$, none occurring in $\mathcal{D}$. Let $\mathcal{D}_{0}^{\$}=$ the result of surgery on $\mathcal{D}_{0}$ at $s_{1}, \ldots, s_{m}$ using $v_{1}, \ldots, v_{m}$. For each $i \in(m)$ fix $\theta_{i}$ such that $\mathbf{0} \theta_{i}$ is the m-formula label of $s_{i}$ in $\mathcal{D}_{0}$, and let $\mathcal{D}_{i}^{\prime}$ be the subdeduction of $\mathcal{D}_{0}$ with root at $s_{i}$. Consider any $i \in(m)$. Since $s_{i}$ is open in $\mathcal{D}_{0}, A\left(\mathcal{D}_{i}^{\prime}\right) \subseteq A\left(\mathcal{D}_{0}\right)$; so $\mathcal{M}, u, v \Vdash A\left(\mathcal{D}_{i}^{\prime}\right)$. Since $h t\left(\mathcal{D}_{i}^{\prime}\right) \leq h t\left(\mathcal{D}_{0}\right)$, by the $\operatorname{IH}\left\langle A\left(\mathcal{D}_{i}^{\prime}\right), \mathbf{0} \theta_{i}\right\rangle$ is $\mathcal{M}$-valid at $u$. So $\mathcal{M}, u, v \Vdash \mathbf{0} \theta_{i}$. So

\footnotetext{
${ }^{26}$ If there is no such $v$, vacuously $\mathcal{M}, u \models \square \varphi$.
} 
$\mathcal{M}, u \models \mathbf{0}^{-1} A\left(\mathcal{D}_{0}^{\$}\right)$. Consider any $v^{\prime}$ so that $u R^{+} v^{\prime} ;{ }^{27}$ fix a $u^{\prime}$ so that $u \sqsubseteq u^{\prime} R v^{\prime}$. As in the previous case,

$$
A\left(\mathcal{D}_{0}^{\$}\right) \subseteq\left(A\left(\mathcal{D}_{0}\right) \cap \mathbf{0} F m l\right) \cup\left\{\mathbf{0} \theta_{i \in(m)}\right\} \cup\{\mathbf{1} \top\} .
$$

By the Persistence Lemma $\mathcal{M}, u^{\prime} \models \mathbf{0}^{-1} A\left(\mathcal{D}_{0}^{\$}\right)$. Since $\mathcal{M}, v^{\prime} \models \top, \mathcal{M}, u^{\prime}, v^{\prime} \Vdash \mathbf{1} \top$. So $\mathcal{M}, u^{\prime}, v^{\prime} \Vdash A\left(\mathcal{D}_{0}^{\$}\right)$. Since $h t\left(\mathcal{D}_{0}^{\$}\right) \leq h t\left(\mathcal{D}_{0}\right) \leq n$, by the $\operatorname{IH}\left\langle A\left(\mathcal{D}_{0}^{\$}\right), \mathbf{1} \varphi\right\rangle$ is $\mathcal{M}$ valid at $u^{\prime}$. So $\mathcal{M}, u^{\prime}, v^{\prime} \Vdash \mathbf{1} \varphi$; so $\mathcal{M}, v^{\prime} \models \varphi$. Thus $\mathcal{M}, u \models \square \varphi$; so $\mathcal{M}, u, v \Vdash$ $\mathbf{0} \square \varphi$. (V2) follows, yielding (!).

Assume that [ ] was entered into $\mathcal{D}$ by $\square E$, as pictured in 3.8; recall: $C_{0} \Rightarrow$ $\mathcal{D}_{0}: \mathbf{0} \square \varphi, C_{1} \Rightarrow \mathcal{D}_{1}: \mathbf{1} \theta$. By the $\mathrm{IH},\left(^{*}\right)\left\langle A\left(\mathcal{D}_{0}\right), \mathbf{0} \square \varphi\right\rangle$ and $\left({ }^{* *}\right)\left\langle A\left(\mathcal{D}_{1}\right), \mathbf{1} \theta\right\rangle$ are $\mathcal{M}$ valid at $u$. Assume $(\mathrm{A})$. Since $A\left(\mathcal{D}_{1}\right) \subseteq A(\mathcal{D})$, by (**) $\mathcal{M}, u \Vdash \mathbf{1} \theta$, a contradiction that vacuously yields (V1). Assume $(\mathrm{B})$. Since $A\left(\mathcal{D}_{0}\right) \subseteq A(\mathcal{D})$, by (*) $\mathcal{M}, u, v \Vdash$ $\mathbf{0} \square \varphi$; so $\mathcal{M}, u \models \square \varphi$. Since $u \sqsubseteq u, R^{+} v$, and so $\mathcal{M}, v \models \varphi$. So $\mathcal{M}, u, v \Vdash \mathbf{1} \varphi$. (V2) follows, yielding (!).

Assume that [ ] was entered into $\mathcal{D}$ by $\diamond E^{+}$, as pictured in 3.8. By the $\mathrm{IH},\left(^{*}\right)$ $\left\langle A\left(\mathcal{D}_{0}\right), \mathbf{0} \diamond \top\right\rangle$ is $\mathcal{M}$-valid at $u$. Assume $(\mathrm{A})$. By $\left(^{*}\right) \mathcal{M}, u \Vdash \mathbf{0} \diamond \top$, for a contradiction; (V1) follows. Assume (B). Since $\mathcal{M}, v \models \top, \mathcal{M}, u, v \Vdash 1 \top$. (V2) follows, yielding (!). Note: this is the only place in this proof in which the difference between $\mathcal{M}$-validity at $u$ and $\mathcal{M}$-validity ${ }^{-}$at $u$ matters. ${ }^{28}$

By induction, for any deduction $\mathcal{D}$ with conclusion $\chi,\langle A(\mathcal{D}), \chi\rangle$ is $\mathcal{M}$-valid at $u$. Thus for any deduction $\mathcal{D}$ with conclusion $\chi,\langle A(\mathcal{D}), \chi\rangle$ is $\mathcal{M}$-valid. Thus for any deduction $\mathcal{D}$ with conclusion $\chi,\langle A(\mathcal{D}), \chi\rangle$ is IK-valid.

\subsection{Observation}

$\vdash_{I K}^{-}$is sound with respect to IK-validity ${ }^{-}$. Proof is a slight variation of that for 6.1 . (In verifying $\left(\mathrm{V}^{-}\right)$, don't assume that $u$ is a dead-end. Of course we omit the last induction case, for $\diamond E^{+}$.)

\subsection{Observation}

$\vdash^{-} \varsubsetneqq \vdash$. Proof. The inclusion is trivial. Trivially $\mathbf{0} \diamond \top \vdash \mathbf{1} \top$. Let $W=1$, $\{\langle 0,0\rangle\}$, and $0 R 0 ; F=\langle W, R$, $\bigsqcup\rangle$ is an IK-frame. For any valuation function $\mathcal{V}$ on $W \times S,\langle\mathbf{0} \diamond \top, \mathbf{1} \top\rangle$ is not $\langle F, \mathcal{V}\rangle$-valid ${ }^{-}$at 0 , this because $\langle F, \mathcal{V}\rangle, 0 \Vdash \mathbf{0} \diamond \top$ but $\langle F, \mathcal{V}\rangle, 0 \nVdash \mathbf{1} \top$, violating $\left(\mathrm{V}^{-}{ }^{-}\right)$. By 6.2, $\mathbf{0} \diamond \top \nvdash^{-} \mathbf{1} \top$.

\subsection{Remark}

One might think that by restricting our attention to $\diamond$-free formulas, we could dispense with right-completeness in our definition of being an IK-frame; after all,

\footnotetext{
${ }^{27}$ If there is no such $v^{\prime}$, vacuously $\mathcal{M}, u \models \square \varphi$.

${ }^{28}$ We needed " $u$ is a dead-end" in (A) to make use of the IH.
} 
the proof of the Persistence Lemma would then not need that IK-frames are rightcomplete. But that is not the case: we needed the right-completeness of IK-frames to handle $\mathbf{0} \supset I$.

Example. Let $\sqsubseteq$ be reflexive on $3,0 \sqsubseteq 2,0 R 1$, and let $F=\langle 3$,,$R\rangle$; for $\pi \in S$, let $\mathcal{V}(u, \pi)=0$ for all $u \in 3$, and let $\mathcal{M}=\langle F, \mathcal{V}\rangle . F$ is not an IK-frame, since it is not right-complete. Suppose that we were to consider it one, and so consider $\mathcal{M}$ an IK-model. Check that $\mathbf{1} \neg \pi \vdash_{I K} \mathbf{0} \neg \square \pi$. But $\langle\{\mathbf{1} \neg \pi\}, \mathbf{0} \neg \square \pi\rangle$ would not be $\mathcal{M}$-valid at 0 , since $\mathcal{M}, 1 \models \neg \pi$, but $\mathcal{M}, 2 \models \square \pi$.

\section{Canonical Models and the Completeness of IK}

\subsection{Definitions}

Until further notice, let $\vdash$ be any monotonic finitary consequence relation ${ }^{29}$ on $M F m l$ such that $\vdash_{I K} \subseteq \vdash$.

Definitions For $\Sigma \subseteq F m l, \Sigma$ is $\vee$-complete iff for any $\varphi_{0}, \varphi_{1} \in F m l$, if $\left(\varphi_{0} \vee\right.$ $\left.\varphi_{1}\right) \in \Sigma$ then for some $i \in 2 \varphi_{i} \in \Sigma$.

Consider a $\Gamma \subseteq M F m l$. $\Gamma$ is $\vee$-complete iff for both markers $\boldsymbol{m}$, if $\boldsymbol{m}\left(\varphi_{0} \vee \varphi_{1}\right) \in \Gamma$ then for some $i \in 2 \boldsymbol{m} \varphi_{i} \in \Gamma$.

For a marker $\boldsymbol{m}$ let $\Gamma$ be $\boldsymbol{m}$-closed under $\vdash$ iff for every $\varphi \in F m l$ if $\Gamma \vdash \boldsymbol{m} \varphi$ then $\boldsymbol{m} \varphi \in \Gamma$. $\Gamma$ is closed under $\vdash$ iff $\Gamma$ is $\boldsymbol{m}$-closed under $\vdash$ for both markers $\boldsymbol{m}$. $\Gamma$ is $\vee$ complete iff for both markers $\boldsymbol{m}$, if $\boldsymbol{m}\left(\varphi_{0} \vee \varphi_{1}\right) \in \Gamma$ then for some $i \in 2 \boldsymbol{m} \varphi_{i} \in \Gamma$. Hereafter let 'closed $\vdash$ ' abbreviate 'closed under $\vdash$ '.

\subsection{Definitions}

Let $W_{\vdash}=\{\Sigma \subseteq F m l \mid \Sigma$ is $\vee$-complete, $\mathbf{0} \Sigma$ is $\mathbf{0}$-closed under $\vdash$, and $\perp \notin \Sigma\}$.

Consider $\Sigma_{0}, \Sigma_{1} \subseteq F m l$. Let $\Sigma_{0} R_{\vdash} \Sigma_{1}$ iff $\Sigma_{0}, \Sigma_{1} \in W_{\vdash}, \square^{-1} \Sigma_{0} \subseteq \Sigma_{1}$ and $\diamond \Sigma_{1} \subseteq \Sigma_{0}$.

$W_{\vdash}$ and $R_{\vdash}$ are the canonical world-domain and accessibility relation under $\vdash$. For the rest of this section I will mostly omit the subscript for $\vdash$.

\subsection{Observation}

If $\Sigma \in W$ then $\top \in \Sigma$, and so $\Sigma \neq\{\}$. Proof. Assume that $\Sigma \in W$. Since \{\}$\vdash \mathbf{0} \top$, $\mathbf{0} \Sigma \vdash \mathbf{0} \top$; since $\mathbf{0} \Sigma$ is $\mathbf{0 I K}$-closed, $\mathbf{0} \top \in \mathbf{0} \Sigma$. So $\top \in \Sigma$.

\subsection{Definition}

For $\chi \in M F m l, \Gamma$ avoids $\chi$ under $\vdash$ iff: $\Gamma$ is closed under $\vdash$ and $\vee$-complete, and $\chi \notin \Gamma$.

\footnotetext{
${ }^{29}$ See section 5.6
} 


\section{5 $0 \perp$-Avoidance Lemma for $\vdash$}

For any $\Sigma_{0}, \Sigma_{1} \subseteq F m l, \mathbf{0} \Sigma_{0} \cup \mathbf{1} \Sigma_{1}$ avoids $\mathbf{0} \perp$ iff $\Sigma_{0} \in W$ and either $\Sigma_{1}=\{\}$ or $\Sigma_{0} R \Sigma_{1}$.

Proof. Let $\Gamma=\mathbf{0} \Sigma_{0} \cup \mathbf{1} \Sigma_{1}$. Assume the left-side. Since $\Gamma$ is $\vee$-complete, $\Sigma_{0}$ is $\vee$-complete. Since $\Gamma$ is $\mathbf{0}$-closed $\vdash_{\vdash}, \mathbf{0} \Sigma_{0}$ is. If $\perp \in \Sigma_{0}$ then $\mathbf{0} \perp \in \Gamma$, contrary to the left-side. Thus $\Sigma_{0} \in W$. Assume that $\Sigma_{1} \neq\{\}$; fix a $\psi \in \Sigma_{1}$. Since $\Gamma$ is $\vee$-complete, $\Sigma_{1}$ is. Assume that $\mathbf{0} \Sigma_{1} \vdash \mathbf{0} \varphi$. Fix $\varphi_{1}, \ldots, \varphi_{m} \in \Sigma_{1}$ so that $\left\{\mathbf{0} \varphi_{i \in(m)}\right\} \vdash \mathbf{0} \varphi$. Assume that $m=0$. Using $\operatorname{Transfer}_{0}, \mathbf{1} \psi \vdash \mathbf{1} \varphi$; since $\mathbf{1} \psi \in \Gamma, \mathbf{1} \varphi \in \Gamma$. Assume that $m>0$. Using Transfer $m,\left\{\mathbf{1} \varphi_{i \in(m)}\right\} \vdash \mathbf{1} \varphi$. Since $\left\{\mathbf{1} \varphi_{i \in(m)}\right\} \subseteq \Gamma$ and $\Gamma$ is $\mathbf{1}$-closed $_{\vdash}, \mathbf{1} \varphi \in \Gamma$. Either way, $\varphi \in \Sigma_{1}$. Thus $\mathbf{0} \Sigma_{1}$ is $\mathbf{0}$-closed $\vdash$. If $\perp \in \Sigma_{1}$ then $\mathbf{1} \perp \in \Gamma$; using $\mathbf{1} \perp E_{0}$, $\Gamma \vdash \mathbf{0} \perp$; since $\Gamma$ is $\mathbf{0}$-closed $\vdash, \mathbf{0} \perp \in \Gamma$, a contradiction. Thus $\Sigma_{1} \in W$. Assume that $\theta \in \Sigma_{1}$. So $\mathbf{1} \theta \in \Gamma$; since $\mathbf{1} \theta \vdash \mathbf{0} \diamond \theta$ and $\Gamma$ is $\mathbf{0}$-closed $\vdash, \mathbf{0} \diamond \theta \in \Gamma$; so $\diamond \theta \in \Sigma_{0}$. Thus $\diamond \Sigma_{1} \subseteq \Sigma_{0}$. Assume that $\varphi \in \square^{-1} \Sigma_{0} ;$ so $\mathbf{0} \square \varphi, \mathbf{1} \psi \in \Gamma$. Since $\{\mathbf{0} \square \varphi, \mathbf{1} \psi\} \vdash \mathbf{1} \varphi$ and $\Gamma$ is $\mathbf{1}$-closed, $1 \varphi \in \Gamma$; so $\varphi \in \Sigma_{1}$. Thus $\square^{-1} \Sigma_{0} \subseteq \Sigma_{1}$. We have shown that $\Sigma_{0} R \Sigma_{1}$.

Assume the right-side. If $\Sigma_{1}=\{\}, \Gamma=\mathbf{0} \Sigma_{0}$; so $\Gamma$ avoids $\mathbf{0} \perp$. Assume that $\Sigma_{0} R \Sigma_{1}$. So $\Sigma_{1} \in W$. Claim 1: $\Gamma$ is $\mathbf{0}$-closed $\vdash$. Assume that $\Gamma \vdash \mathbf{0} \theta$. Since $\vdash$ is finitary we may fix $\psi_{i \in(m)} \in \Sigma_{1}$ so that $\mathbf{0} \Sigma_{0} \cup\left\{\mathbf{1} \psi_{i \in(m)}\right\} \vdash \mathbf{0} \theta$. Since $\Sigma_{1} \in W$, $\Sigma_{1}$ is closed under conjunction. Let $\psi$ be $\bigwedge_{i \in(m)} \psi_{i}$. So $\mathbf{0} \Sigma_{1} \vdash \mathbf{0} \psi$; since $\Sigma_{1} \in W$, $\mathbf{0} \psi \in \mathbf{0} \Sigma_{1}$; so $\psi \in \Sigma_{1}$. Since $\Sigma_{0} R \Sigma_{1}, \diamond \psi \in \Sigma_{0}$; so $\mathbf{0} \diamond \psi \in \mathbf{0} \Sigma_{0}$. Also, using $\mathbf{1} \& E, \mathbf{0} \Sigma_{0} \cup\{\mathbf{1} \psi\} \vdash \mathbf{0} \theta$, witnessed by a deduction in which for some $v \in \operatorname{Var}\{\}$ is a barrier with exception for $v: \mathbf{1} \psi$. Using $\diamond E, \mathbf{0} \Sigma_{0} \cup\{\mathbf{0} \diamond \psi\} \vdash \mathbf{0} \theta$; so $\mathbf{0} \Sigma_{0} \vdash \mathbf{0} \theta$. Since $\Sigma_{0}$ is $\mathbf{0}$-closed ${ }_{\vdash}, \mathbf{0} \theta \in \mathbf{0} \Sigma_{0}$. Claim 1 follows. Claim 2: $\Gamma$ is $\mathbf{1}$-closed $d_{\vdash}$. Assume that $\Gamma \vdash \mathbf{1} \theta$. Fix $\psi \in \Sigma_{1}$ so that $\mathbf{0} \Sigma_{0} \cup\{\mathbf{1} \psi\} \vdash \mathbf{1} \theta$. So $\mathbf{0} \Sigma_{0} \cup\{\mathbf{1} \psi\} \vdash \mathbf{1} \theta$; using $\mathbf{1} \supset I, \mathbf{0} \Sigma_{0} \cup\{\mathbf{1} \top\} \vdash \mathbf{1}(\psi \supset \theta)$, witnessed by a deduction in which for some $v \in \operatorname{Var}\{\}$ is a barrier with exception for $v: \mathbf{1} \top$. Using $\square I, \mathbf{0} \Sigma_{0} \vdash \mathbf{0} \square(\psi \supset \theta)$. Since $\Sigma_{0}$ is $\mathbf{0}$-closed $\vdash, \mathbf{0} \square(\psi \supset \theta) \in \mathbf{0} \Sigma_{0}$; so $\square(\psi \supset \theta) \in \Sigma_{0}$. So $(\psi \supset \theta) \in \Sigma_{1}$; so $\mathbf{0}(\psi \supset \theta) \in \mathbf{0} \Sigma_{1}$. Note that $\mathbf{0} \Sigma_{1} \vdash \mathbf{0} \psi$; so $\mathbf{0} \Sigma_{1} \vdash \mathbf{0} \theta$. Since $\Sigma_{1}$ is $\mathbf{0}$-closed $\mathbf{0} \theta \in \mathbf{0} \Sigma_{1}$. So $\theta \in \Sigma_{1}$; so $\mathbf{1} \theta \in \Gamma$. Claim 2 follows. Claim 3: $\Gamma$ is $\vee$-complete. Assume that $\mathbf{0}\left(\varphi_{0} \vee \varphi_{1}\right) \in \Gamma$. So $\left(\varphi_{0} \vee \varphi_{1}\right) \in \Sigma_{0}$; fixing an $i \in 2$ so that $\varphi_{i} \in \Sigma_{0}$, $\mathbf{0} \varphi_{i} \in \Gamma$. Similarly, assuming that $\mathbf{1}\left(\varphi_{0} \vee \varphi_{1}\right) \in \Gamma$, we may fix $i \in 2$ so that $\varphi_{i} \in \Sigma_{1}$, and so $\mathbf{1} \varphi_{i} \in \Gamma$. Claim 3 follows. If $\mathbf{0} \perp \in \Gamma, \perp \in \Sigma_{0}$ for a contradiction; so $\mathbf{0} \perp \notin \Gamma$. Thus $\Gamma$ avoids $\mathbf{0} \perp$.

\subsection{Convention}

For the rest of this section, let $\left\langle\zeta_{i}\right\rangle_{i \in \omega}$ list the members of $F m l$ so that every member occurs infinitely often on the list; call the latter condition "infinite visitation".

\subsection{The Avoidance Theorem for $\vdash$}

Consider any $\Gamma \subseteq M F m l$ and $\chi \in M F m l$ such that $\Gamma \nvdash \chi$. There is a $\Sigma_{0} \in W$ and a $\Sigma_{1} \subseteq F m l$ such that $\Gamma \subseteq \mathbf{0} \Sigma_{0} \cup \mathbf{1} \Sigma_{1}, \mathbf{0} \Sigma_{0} \cup \mathbf{1} \Sigma_{1}$ avoids $\chi$, and furthermore: (a) if $\Gamma \cup\{\mathbf{1} \top\} \vdash \chi$ then $\Sigma_{1}=\{\}$; (b) otherwise $\Sigma_{0} R \Sigma_{1}$. 
Proof. Let

$$
\Gamma^{\prime}= \begin{cases}\Gamma & \text { if } \Gamma \cup\{\mathbf{1} \top\} \vdash \chi \\ \Gamma \cup\{\mathbf{1} \top\} & \text { otherwise. }\end{cases}
$$

We will construct a $q \in \omega+1$ and a "double sequence" $\left\langle\Phi_{j}, \Psi_{j}\right\rangle_{j \in q}$, and prove that $q=\omega$. For each $j \in q$ we will have $\Phi_{j}, \Psi_{j} \subseteq F m l$.

Let $0 \in q$ and $\Phi_{0}=\Psi_{0}=\{\}$. Given $j \in \omega$, assume that $j \in q$ and for some $n \in \omega, j \in[4 n, 4 n+3]$. Fix that $n$; so $4 n \in q$. Let $4 n+1 \in q$ and $\Psi_{4 n+1}=$ $\Psi_{4 n}$. If $\Gamma^{\prime} \cup \mathbf{0} \Phi_{4 n} \cup \mathbf{1} \Psi_{4 n} \nvdash \mathbf{0} \zeta_{n}$, let $4 n+2 \in q, \Phi_{4 n+2}=\Phi_{4 n+1}=\Phi_{4 n}$ and $\Psi_{4 n+2}=\Psi_{4 n+1}$. Assume that $\Gamma^{\prime} \cup \mathbf{0} \Phi_{4 n} \cup \mathbf{1} \Psi_{4 n} \vdash \mathbf{0} \zeta_{n}$. Let $\Phi_{4 n+1}=\Phi_{4 n} \cup\left\{\zeta_{n}\right\}$. If $\zeta_{n}$ is not a disjunction let $4 n+2 \in q, \Phi_{4 n+2}=\Phi_{4 n+1}$ and $\Psi_{4 n+2}=\Psi_{4 n+1}$. Assume that $\zeta_{n}$ is $\left(\varphi_{0} \vee \varphi_{1}\right)$. If for some $i \in 2 \Gamma^{\prime} \cup \mathbf{0} \Phi_{4 n+1} \cup \mathbf{1} \Psi_{4 n+1} \cup\left\{\mathbf{0} \varphi_{i}\right\} \nvdash \chi$, fix such an $i$ and let $4 n+2 \in q, \Phi_{4 n+2}=\Phi_{4 n+1} \cup\left\{\varphi_{i}\right\}$, and $\Psi_{4 n+2}=\Psi_{4 n+1}$. Otherwise (the bad case for $4 n+2$ ) let $q=4 n+2$, and we are done. Now assume that $4 n+2 \in q$ (and so the bad case for $4 n+2$ did not obtain). Let $4 n+3 \in q$ and $\Phi_{4 n+3}=\Phi_{4 n+2}$. If $\Gamma^{\prime} \cup \mathbf{0} \Phi_{4 n+2} \cup \mathbf{1} \Psi_{4 n+2} \nvdash \mathbf{1} \zeta_{n}$, let $\Phi_{4 n+3}=\Phi_{4 n+4}=\Phi_{4 n+2}$. Assume that $\Gamma^{\prime} \cup \mathbf{0} \Phi_{4 n+2} \cup \mathbf{1} \Psi_{4 n+2} \vdash \mathbf{1} \zeta_{n}$. Let $\Psi_{4 n+3}=\Psi_{4 n+2} \cup\left\{\zeta_{n}\right\}$. If $\zeta_{n}$ is not a disjunction let $4 n+4 \in q, \Psi_{4 n+4}=\Psi_{4 n+3}$ and $\Phi_{4 n+4}=\Phi_{4 n+3}$. Assume that $\zeta_{n}$ is $\left(\varphi_{0} \vee \varphi_{1}\right)$. If for some $i \in 2 \Gamma^{\prime} \cup \mathbf{0} \Phi_{4 n+2} \cup \mathbf{1} \Psi_{4 n+2} \cup\left\{\mathbf{1} \varphi_{i}\right\} \nvdash \chi$, fix such an $i$; let $4 n+4 \in q, \Psi_{4 n+4}=\Psi_{4 n+1} \cup\left\{\varphi_{i}\right\}$ and $\Phi_{4 n+4}=\Phi_{4 n+3}$. Otherwise (the bad case for $4 n+4$ ) let $q=4 n+4$, and we are done.

Claim 1: for every $j \in \omega$, (i) $j \in q$, (ii) $\Gamma^{\prime} \cup \mathbf{0} \Phi_{j} \cup \mathbf{1} \Psi_{j} \nvdash \chi$, (iii) if $j>0$ and $j$ is even then the bad case for $j$ does not obtain. Proof by induction on $j$.

The base-step. 0 satisfies (i) by stipulation, (ii) by assumption, and (iii) vacuously.

The induction step. Given $j$, assume the obvious IH. Fix $n \in \omega$ so that $4 n \leq j \leq$ $4 n+3$. Case 1: $j=4 n$. So $4 n+1 \in q$, and $j+1$ satisfies (i). If $\Gamma^{\prime} \cup \mathbf{0} \Phi_{4 n} \cup$ $\mathbf{1} \Psi_{4 n} \nvdash \mathbf{0} \zeta_{n}$, the IH implies that $j+1$ satisifes (ii)-(iii), and so $j+2$ satisfies (i)(iii). Assume that $\Gamma^{\prime} \cup \mathbf{0} \Phi_{4 n} \cup \mathbf{1} \Psi_{4 n} \vdash \mathbf{0} \zeta_{n}$. If $\Gamma^{\prime} \cup \mathbf{0} \Phi_{4 n+1} \cup \mathbf{1} \Psi_{4 n+1} \vdash \chi$, cutting $\mathbf{0} \zeta_{n}$ shows that $\Gamma^{\prime} \cup \mathbf{0} \Phi_{4 n} \cup \mathbf{1} \Psi_{4 n} \vdash \chi$, contrary to the IH. So $4 n+1$ satisfies (ii), and vacuously satisfies (iii). Case $2: j=4 n+1$. If $\zeta_{n}$ is not a disjunction, clearly $4 n+2$ satisfies (i)-(iii). Assume that $\zeta_{n}$ is $\left(\varphi_{0} \vee \varphi_{1}\right)$. Assume that for both $i \in 2$ $\Gamma^{\prime} \cup \mathbf{0} \Phi_{4 n+1} \cup \mathbf{1} \Psi_{4 n+1} \cup\left\{\mathbf{0} \varphi_{i}\right\} \vdash \chi$. Since

$$
\Gamma^{\prime} \cup \mathbf{0} \Phi_{4 n+1} \cup \mathbf{1} \Psi_{4 n+1} \cup\left\{\mathbf{0}\left(\varphi_{0} \vee \varphi_{1}\right)\right\}=\Gamma^{\prime} \cup \mathbf{0} \Phi_{4 n} \cup \mathbf{1} \Psi_{4 n} \cup\left\{\mathbf{0} \zeta_{n}\right\},
$$

using $\mathbf{0} \vee E$ gives a witness that $\Gamma^{\prime} \cup \mathbf{0} \Phi_{4 n} \cup \mathbf{1} \Psi_{4 n} \cup\{\mathbf{0} \zeta\} \vdash \chi$, contrary to our IH. So $4 n+2$ satisfies (i)-(iii). Similar arguments for $j=4 n+3$ and $j=4 n+4$ show that such $j+1$ satisfy (i)-(iii). Claim 1 follows. Thus $q=\omega$.

Let $\Sigma_{0}=\bigcup_{j \in \omega} \Phi_{j}$ and $\Sigma_{1}=\bigcup_{j \in \omega} \Psi_{j}$. Let $\Gamma^{*}=\mathbf{0} \Sigma_{0} \cup \mathbf{1} \Sigma_{1}$. Clearly $\Gamma^{\prime} \subseteq \Gamma^{*}$. Assume that $\chi \in \Gamma^{*}$. By the infinite visitation condition there is an $n$ so that either $\zeta_{n}$ is $\mathbf{0}^{-1} \chi$ and $\chi \in \mathbf{0} \Phi_{4 n+1}$, violating Claim 1.(ii) for $4 n+1$, or $\zeta_{n}$ is $\mathbf{1}^{-1} \chi$ and $\chi \in \mathbf{1} \Psi_{4 n+3}$, violating Claim 1.(ii) for $4 n+3$. So $\chi \notin \Gamma^{*}$.

Claim 2: $\Gamma^{*}$ is closed $\vdash$. Given $\theta \in F m l$, assume that $\Gamma^{*} \vdash \mathbf{0} \theta$. By the infinite visitation condition we may fix an $n \in \omega$ so that $\theta$ is $\zeta_{n}$ and $\Gamma^{\prime} \cup \mathbf{0} \Phi_{4 n} \cup \mathbf{1} \Psi_{4 n} \vdash \mathbf{0} \theta$. So $\theta \in \Phi_{4 n+1}$; so $\theta \in \Sigma_{0}$; so $\mathbf{0} \theta \in \Gamma^{*}$. Thus $\mathbf{0} \Gamma^{*}$ is $\mathbf{0}$-closed $\vdash$. A similar argument shows that $\mathbf{1} \Gamma^{*}$ is $\mathbf{1}$-closed ${ }_{\vdash}$. Claim 2 follows.

Claim 3: $\Gamma^{*}$ is $\vee$-complete. Consider any $\varphi_{0}, \varphi_{1} \in F m l$. Assume that $\mathbf{0}\left(\varphi_{0} \vee \varphi_{1}\right) \in$ $\Gamma^{*}$. By the infinte visitation condition we may fix an $n \in \omega$ so that $\left(\varphi_{0} \vee \varphi_{1}\right)$ is $\zeta_{n}$ 
and $\Gamma^{\prime} \cup \mathbf{0} \Phi_{4 n} \cup \mathbf{1} \Psi_{4 n} \vdash \mathbf{0}\left(\varphi_{0} \vee \varphi_{1}\right)$. So for some $i \in 2 \varphi_{i} \in \Phi_{4 n+2}$; so $\varphi_{i} \in \Sigma_{0}$; so $\mathbf{0} \varphi_{i} \in \Gamma^{*}$. If $\mathbf{1}\left(\varphi_{0} \vee \varphi_{1}\right) \in \Gamma^{*}$, a similar argument shows that for some $i \in 2$ $1 \varphi_{i} \in \Gamma^{*}$. Claim 3 follows. So $\Gamma^{*}$ avoids $\chi$.

Claim 4: $\Gamma^{*}$ avoids $\mathbf{0} \perp$. If $\mathbf{0} \perp \in \Gamma^{*}$ then $\Gamma^{*} \vdash \chi$ using $\mathbf{0} \perp E$, and then $\chi \in \Gamma^{*}$, since $\Gamma^{*}$ is closed ${ }_{\vdash}$, for a contradiction.

By the $\mathbf{0} \perp$-Avoidance Lemma, $(*)$ either $\Sigma_{1}=\{\}$ or $\Sigma_{0} R \Sigma_{1}$. Assume that $\Gamma \cup$ $\{1 \top\} \vdash \chi$; so $1 \top \notin \Gamma^{*}$; if $\Sigma_{1} \neq\{\}$ then $\Sigma_{1} \in W$, and so $\top \in \Sigma_{1}$, a contradiction. So $\Sigma_{1}=\{\}$. Assume that $\Gamma \cup\{\mathbf{1} T\} \nvdash \chi$; because we started with $\Gamma^{\prime}$ in the above construction, $1 \top \in \Gamma^{*}$. So $\top \in \Sigma_{1}$; so $\Sigma_{1} \neq\{\}$; so $\Sigma_{0} R \Sigma_{1}$.

\subsection{Lindenbaum's Lemma for $\vdash$}

If $\Phi \subseteq F m l, \psi \in F m l$, and $\mathbf{0} \Phi \nvdash \mathbf{0} \psi$, then for some $\Sigma \in W, \psi \notin \Sigma$ and $\Phi \subseteq \Sigma$.

Proof. Assume the if-clause. Applying the Avoidance Theorem, with $\Gamma=\mathbf{0} \Phi$ and taking $\chi$ to be $\mathbf{0} \psi$, we may fix $\Sigma_{0}$ and $\Sigma_{1}$ so that $\mathbf{0} \Phi \subseteq \mathbf{0} \Sigma_{0} \cup \mathbf{1} \Sigma_{1}, \Sigma_{0} \in W$, and $\mathbf{0} \psi \notin \mathbf{0} \Sigma_{0}$, which yields $\psi \notin \Sigma_{0}$.

\subsection{Definition}

Let $\sqsubseteq \vdash=\subseteq \mid W_{\vdash}$. Let $F_{\vdash}=\left\langle W_{\vdash}, R_{\vdash}, \sqsubseteq \vdash\right\rangle ; F_{\vdash}$ is the canonical frame for $\vdash$. In this section I will usually omit the subscript.

\subsection{Remark}

The proofs of the next two lemmas are "one-sided" cousins of the proof of the Avoidance Theorem.

\subsection{The Unbox Lemma for $\vdash$}

If $\Sigma \subseteq F m l, \Psi \in W, \mathbf{0} \Sigma$ is $\mathbf{0}$-closed , $_{-}$and $\square^{-1} \Sigma \subseteq \Psi$, then for some $\Phi, \Sigma \subseteq \Phi$ and $\Phi R \Psi$.

Proof. Assume the if-clause. Let $\Gamma=\mathbf{0} \Sigma \cup \mathbf{1} \Psi$. We will define a $q \in \omega+1$ and a sequence $\left\langle\Phi_{j}\right\rangle_{j \in q}$ as follows. Let $0 \in q$ and $\Phi_{0}=\{\}$. Assume that $2 n \in q$. Let $2 n+1 \in q$. If $\Gamma \cup \mathbf{0} \Phi_{2 n} \nvdash \mathbf{0} \zeta_{n}$ let $\Phi_{2 n+2}=\Phi_{2 n+1}=\Phi_{2 n}$. Assume that $\Gamma \cup \mathbf{0} \Phi_{2 n} \vdash$ $\mathbf{0} \zeta_{n}$. Let $\Phi_{2 n+1}=\Phi_{2 n} \cup\left\{\zeta_{n}\right\}$, and if $\zeta_{n}$ is not a disjunction let $\Phi_{2 n+2}=\Phi_{2 n+1}$. Assume that $\zeta_{n}$ is $\left(\varphi_{0} \vee \varphi_{1}\right)$. If for some $i \in 2$,

$\left(*_{2 n+2}\right)$ for every $\sigma \in F m l$, if $\Gamma \cup \mathbf{0} \Phi_{2 n+1} \cup\left\{\mathbf{0} \varphi_{i}\right\} \vdash \mathbf{0} \square \sigma$ then $\sigma \in \Psi$,

fix such an $i$ and let $\Phi_{2 n+2}=\Phi_{2 n+1} \cup\left\{\varphi_{i}\right\}$. If neither $i \in 2$ satisfies condition $\left(*^{*} n+2\right)$ (the bad case for $\left.2 n+2\right)$, let $q=2 n+2$.

Claim 1: for every $j \in \omega$, (i) $j \in q$; (ii) $\Gamma \cup \mathbf{0}_{j}$ is $\mathbf{1}$-closed ${ }_{\vdash}$; (iii) for every $\sigma \in F m l$, if $\Gamma \cup \mathbf{0} \Phi_{j} \vdash \mathbf{0} \square \sigma$ then $\sigma \in \Psi$; (iv) if $j>0$ and $j$ is even, the bad case for $j$ does not obtain. Proof is by induction.

Base step. 0 satisfies (i) by stipulation. Given $\delta \in F m l$, assume that $\Gamma \vdash \mathbf{1} \delta$. Since $\vdash$ is finitary and $\Psi$ is closed under conjunction, we may fix $\psi \in \Psi$ so that $\mathbf{0} \Sigma \cup\{\mathbf{1} \psi\} \vdash \mathbf{1} \delta$. So $\mathbf{0} \Sigma \cup\{\mathbf{1} \psi\} \vdash \mathbf{1} \delta$. Using $\mathbf{1} \supset I, \mathbf{0} \Sigma \cup\{\mathbf{1} \top\} \vdash \mathbf{1}(\psi \supset \delta)$, witnessed 
by a deduction $\mathcal{D}$ in which for some $v \in \operatorname{Var}\{\}$ is a barrier with exception for $v: \mathbf{1} \top$. Using $\square I, \mathbf{0} \Sigma \vdash \mathbf{0} \square(\psi \supset \delta)$. So $\square(\psi \supset \delta) \in \Sigma$. Since $\square^{-1} \Sigma \subseteq \Psi$, $(\psi \supset \delta) \in \Psi$; so $\mathbf{0}(\psi \supset \delta) \in \mathbf{0} \Psi$. Since $\Psi \in W, \Psi$ is closed under conjunction; so $\psi \in \Psi$; so $\mathbf{0} \psi \in \mathbf{0} \Psi$. Since $\Psi \in W$, it is $\mathbf{0}$-closed $\vdash$; so $\delta \in \Psi$; so $\mathbf{1} \delta \in \Gamma$. So 0 satisfies (ii). Given $\sigma \in F m l$, assume that $\Gamma \vdash \mathbf{0} \square \sigma$. Now fix $\psi \in \Psi$ so that $\mathbf{0} \Sigma \cup\{\mathbf{1} \psi\} \vdash \mathbf{0} \square \sigma$. As above, $\mathbf{0} \Sigma \cup\{\mathbf{1} \psi\} \vdash \mathbf{0} \square \sigma$, which is witnessed by a deduction in which for some $\nu \in \operatorname{Var}\{\}$ is a barrier with exception for $v: \mathbf{1} \psi$. Using $\diamond E, \mathbf{0} \Sigma \cup\{\mathbf{0} \diamond \psi\} \vdash \mathbf{0} \square \sigma$. So $\mathbf{0} \Sigma \vdash \mathbf{0}(\diamond \psi \supset \square \sigma)$. By 4.2.(4), $\mathbf{0} \Sigma \vdash \mathbf{0} \square(\psi \supset \sigma)$. Since $\Sigma$ is $\mathbf{0}$-closed $\square(\psi \supset \sigma) \in \Sigma$; so $(\psi \supset \sigma) \in \Psi$. Since $\psi \in \Psi, \mathbf{0} \Psi \vdash \boldsymbol{0} \sigma$; so $\sigma \in \Psi$. So 0 satisfies (iii). 0 vacuously satisifes (iv).

Induction step. Given $j$, assume the obvious IH. Fix $n$ so that $2 n \leq j \leq 2 n+1$. Assume that $j=2 n$. So $2 n+1<q$. If $\Gamma \cup \mathbf{0} \Phi_{2 n} \nvdash \zeta_{n}$, the IH insures that $2 n+1$ satisfies (ii)-(iii), it vacuously satisifes (iv), and $2 n+2$ trivially satisifes (i)-(iv). Assume that $\Gamma \cup \mathbf{0} \Phi_{2 n} \vdash \zeta_{n}$. Given $\delta \in F m l$, assume that $\Gamma \cup \mathbf{0} \Phi_{2 n+1} \vdash \mathbf{1} \delta$. Cutting $\mathbf{0} \zeta_{n}, \Gamma \cup \mathbf{0} \Phi_{2 n} \vdash \mathbf{1} \delta$. By the IH $2 n$ satisifes (ii); so $\delta \in \Psi$; so $2 n+1$ satisfies (ii). Similarly, for a given $\sigma \in F m l$ if $\Gamma \cup \mathbf{0} \Phi_{2 n+1} \vdash \mathbf{0} \square \sigma$, cutting $\mathbf{0} \zeta_{n}$ yields $\Gamma \cup \mathbf{0} \Phi_{2 n} \vdash \mathbf{0} \square \sigma$; so by the IH $\sigma \in \Psi$. So $2 n+2$ satisfies (iii). $2 n+1$ vacuously satisfies (iv). If $\zeta_{n}$ is not a disjunction, $2 n+2$ trivially satisfies (i)-(iv). Assume that $\zeta_{n}$ is $\left(\varphi_{0} \vee \varphi_{1}\right)$. Subclaim: for some $i \in 2$, $i$ satisfies $\left(*_{2 n+2}\right)$. Assume not; for both $i \in 2$ fix $\sigma_{i} \in F m l$ so that $\Gamma \cup \mathbf{0} \Phi_{2 n+1} \cup\left\{\mathbf{0} \varphi_{i}\right\} \vdash \mathbf{0} \square \sigma_{i}$ but $\sigma_{i} \notin \Psi$. Let $\sigma$ be $\left(\sigma_{0} \vee \sigma_{1}\right)$. Since $\Psi \neq\{\}, 1 \top \in \Gamma$; so for each $i \in 2$ one use of $\square E$ gives a witness that $\Gamma \cup \mathbf{0} \Phi_{2 n+1} \cup\left\{\mathbf{0} \varphi_{i}\right\} \vdash \mathbf{1} \sigma_{i}$; using $\mathbf{1} \vee I, \Gamma \cup \mathbf{0} \Phi_{2 n+1} \cup\left\{\mathbf{0} \varphi_{i}\right\} \vdash \mathbf{1} \sigma$. Using $1 \vee E, \Gamma \cup \mathbf{0} \Phi_{2 n+1} \vdash \mathbf{1} \sigma$. Cutting $\mathbf{0} \zeta_{n}, \Gamma \cup \mathbf{0} \Phi_{2 n} \vdash \mathbf{1} \sigma$. By the IH, $2 n$ satisifes (ii); so $1 \sigma \in \Gamma \cup \mathbf{0} \Phi_{2 n}$; so $\left(\sigma_{0} \vee \sigma_{1}\right) \in \Psi$. Since $\Psi \in W$, for some $i \in 2 \sigma_{i} \in \Psi$, a contradiction. The subclaim follows. So $2 n+2 \in q$ and $2 n+2$ satisfies (iv). Fix an $i \in 2$ for which $\Phi_{2 n+2}=\Phi_{2 n+1} \cup\left\{\varphi_{i}\right\}$. Given $\delta \in F m l$, assume that $\Gamma \cup \mathbf{0} \Phi_{2 n+2} \vdash \mathbf{1} \delta$. Fix $\theta \in \Psi$ so that $\mathbf{0} \Sigma_{0} \cup\{\mathbf{1} \theta\} \cup \mathbf{0} \Phi_{2 n+2} \vdash \mathbf{1} \delta$. So $\mathbf{0} \Sigma \cup\{\mathbf{1} \theta\} \cup \mathbf{0} \Phi_{2 n+2} \vdash \mathbf{1} \delta$. Using $\mathbf{1} \supset I, \mathbf{0} \Sigma_{0} \cup \mathbf{0} \Phi_{2 n+2} \cup\{\mathbf{1} \top\} \vdash \mathbf{1}(\theta \supset \delta)$, and for some $v$ this is witnessed by a deduction in which \{\} is a barrier with exception for $v: \mathbf{1} \top$. Using $\square I, \mathbf{0} \Sigma \cup \mathbf{0} \Phi_{2 n+2} \vdash \mathbf{0} \square(\theta \supset \delta)$. Since $i$ satisifies $\left(*_{2 n+2}\right),(\theta \supset \delta) \in \Psi$. Since $\mathbf{0} \Psi \vdash \mathbf{0} \theta, \mathbf{0} \Psi \vdash \mathbf{0} \delta$. Since $\Psi$ is $\mathbf{0}$-closed, $\delta \in \Psi$. So $\mathbf{1} \delta \in \Gamma \cup \mathbf{0} \Phi_{2 n+2}$. Thus $2 n+2$ satisfies (ii). Since $i$ satisifies $(* 2 n+2), 2 n+2$ satisfies (iii).

Claim 1 follows by induction. Thus $q=\omega$. Let $\Phi=\bigcup \Phi_{j \in \omega}$. Clearly $\Sigma \subseteq \Phi$.

Claim 2: $\mathbf{0} \Phi \cup \mathbf{1} \Psi$ avoids $\mathbf{0} \perp$. Assume that $\mathbf{0} \Phi \cup \mathbf{1} \Psi \vdash \mathbf{0} \theta$. By the infinite visitation condition we may fix $n \in \omega$ so that $\Gamma \cup \mathbf{0} \Phi_{2 n} \vdash \mathbf{0} \theta$ and $\zeta_{n}$ is $\theta$; so $\zeta_{n} \in \Phi_{2 n+1}$; so $\theta \in \Phi$. So $\mathbf{0} \Phi \cup \mathbf{1} \Psi$ is $\mathbf{0}$-closed $\vdash$. Assume that $\mathbf{0} \Phi \cup \mathbf{1} \Psi \vdash \mathbf{1} \theta$. Since $\vdash$ is finitary we may fix $j \in \omega$ and $\psi \in \Psi$ so that $\mathbf{0} \Sigma \cup\{\mathbf{1} \psi\} \cup \mathbf{0} \Phi_{j} \vdash \mathbf{1} \theta$. Using $\mathbf{1} \& E, \mathbf{0} \Sigma_{0} \cup\{\mathbf{1} \psi\} \cup \mathbf{0} \Phi_{j} \vdash \mathbf{1} \theta$. So $\mathbf{0} \Sigma_{0} \cup\{\mathbf{1} \top\} \cup \mathbf{0} \Phi_{j} \vdash \mathbf{1}(\psi \supset \theta)$, witnessed by a deduction in which, for some $v \in V$ ar, \{\} is a barrier except for $v: \mathbf{1} \top$. Using $\square I, \mathbf{0} \Sigma \cup \mathbf{0} \Phi_{j} \vdash \mathbf{0} \square(\psi \supset \theta)$. By Claim 1(iii), $(\psi \supset \theta) \in \Psi$. Since $\Psi \in W$, $\psi \in \Psi$, and so $\theta \in \Psi$. Thus $\mathbf{0} \Phi \cup \mathbf{1} \Psi$ is $\mathbf{1}$-closed $_{\vdash}$, and so is closed $\mathrm{d}_{\vdash}$. Assume that $\mathbf{0}\left(\varphi_{0} \vee \varphi_{1}\right) \in \mathbf{0} \Phi \cup \mathbf{1} \Psi$; so $\left(\varphi_{0} \vee \varphi_{1}\right) \in \Phi$. Fix $n \in \omega$ so that $\zeta_{n}$ is $\left(\varphi_{0} \vee \varphi_{1}\right)$ and $\left(\varphi_{0} \vee \varphi_{1}\right) \in \Phi_{2 n}$. So $\Gamma \cup \mathbf{0} \Phi_{2 n} \vdash \zeta_{n}$; for some $i \in 2 \varphi_{i} \in \Phi_{2 n+2}$, and so $\varphi_{i} \in \Phi$. Assume that $\mathbf{1}\left(\varphi_{0} \vee \varphi_{1}\right) \in \mathbf{0} \Phi \cup \mathbf{1} \Psi$; so $\left(\varphi_{0} \vee \varphi_{1}\right) \in \Psi$. Since $\Psi \in W$, it is $\vee$-complete; fix $i \in 2$ so that $\varphi_{i} \in \Psi$; so $1 \varphi_{i} \in \mathbf{0} \Phi \cup 1 \Psi$. Thus $\mathbf{0} \Phi \cup 1 \Psi$ is $\vee$-complete. Assume 
that $\mathbf{0} \perp \in \mathbf{0} \Phi \cup \mathbf{1} \Psi$. Using $\mathbf{0} \perp E, \mathbf{0} \Phi \cup \mathbf{1} \Psi \vdash \mathbf{1} \perp$; since $\mathbf{0} \Phi \cup \mathbf{1} \Psi$ is $\mathbf{1}$-closed $\perp \in \Psi$, contrary to $\Psi \in W$. So $\mathbf{0} \perp \notin \mathbf{0} \Phi \cup \mathbf{1} \Psi$. Claim 2 follows.

Since $\Psi \in W, \Psi \neq\{\}$. So by the $\mathbf{0} \perp$-Avoidance Lemma, $\Phi R \Psi$.

\subsection{The Left Lemma for -}

If $\Sigma_{0} R \Sigma_{1} \sqsubseteq \Sigma_{1}^{\prime}$ then for some $\Sigma_{0}^{\prime} \in W \Sigma_{0} \sqsubseteq \Sigma_{0}^{\prime} R \Sigma_{1}^{\prime}$.

Proof. Assume the if-clause. So $\square^{-1} \Sigma_{0} \subseteq \Sigma_{1}^{\prime}$. Take $\Sigma_{0}$ and $\Sigma_{1}^{\prime}$ to be $\Sigma$ and $\Psi$ in the above formulation of the Unbox Lemma. So that lemma yields a $\Sigma_{0}^{\prime}$ (called $\Phi$ in the formulation of the lemma) that is as desired.

\subsection{The Diamond Lemma for $\vdash$}

If $\Phi \in W, \Sigma \subseteq F m l, \Sigma$ is closed under conjunction, and $\diamond \Sigma \subseteq \Phi$, then for some $\Psi, \Phi R \Psi$ and $\Sigma \subseteq \Psi$.

Proof. Assume the if-clause. Let $\Gamma=\mathbf{0} \Phi \cup \mathbf{1} \Sigma$. We will define a $q \in \omega+1$, and a sequence $\left\langle\Psi_{j}\right\rangle_{j \in q}$ as follows. Let $0 \in q$ and $\Psi_{0}=\{\}$. Assume that $2 n \in q$. Let $2 n+1 \in q$. If $\Gamma \cup 1 \Psi_{2 n} \nvdash \mathbf{1} \zeta_{n}$ let $2 n+2 \in q$ and $\Psi_{2 n+2}=\Psi_{2 n+1}=\Psi_{2 n}$. Assume that $\Gamma \cup \mathbf{1} \Psi_{2 n} \vdash \mathbf{1} \zeta_{n}$. Let $\Psi_{2 n+1}=\Psi_{2 n} \cup\left\{\zeta_{n}\right\}$, and if $\zeta_{n}$ is not a disjunction let $2 n+2 \in q$ and $\Psi_{2 n+2}=\Psi_{2 n+1}$. Assume that $\zeta_{n}$ is $\left(\varphi_{0} \vee \varphi_{1}\right)$. If for some $i \in 2$,

$$
(* 2 n+2) \text { for every } \sigma \in \Sigma, \diamond\left(\varphi_{i} \& \sigma \& \wedge \Psi_{2 n+1}\right) \in \Phi,
$$

fix such an $i$ and let $\Psi_{2 n+2}=\Psi_{2 n+1} \cup\left\{\varphi_{i}\right\}$. If neither $i \in 2$ satisfies $\left({ }^{*} 2 n+2\right)$, (the bad case for $2 n+2$ ), let $q=2 n+2$.

Claim 1: for every $j \in \omega$, (i) $j \in q$, (ii) $\Gamma \cup 1 \Psi_{j}$ is 0 IK-closed, (iii) for every $\sigma \in \Sigma, \diamond\left(\sigma \& \wedge \Psi_{j}\right) \in \Phi$, and (iv) if $j>0$ and $j$ is even, the bad case for $j$ does not obtain. Proof is by induction.

Base Step. 0 satisfies (i) by stipulation. Given $\delta \in F m l$, assume that $\Gamma \vdash \mathbf{0} \delta$. Since $\vdash$ is finitary and $\Sigma$ is closed under conjuction, we may fix $\rho \in \Sigma$ so that $\mathbf{0} \Phi \cup\{\mathbf{1} \rho\} \vdash \mathbf{0} \delta$. (If $l=0, \rho$ is $\bigwedge\{\}$ which is $T$.) So $\mathbf{0} \Phi \cup\{\mathbf{1} \rho\} \vdash \mathbf{0} \delta$; this is witnessed by a deduction in which for some $v \in \operatorname{Var}\{\}$ is a barrier with exception for $v: \mathbf{1} \rho$. By one use of $\diamond E, \mathbf{0} \Phi \cup\{\mathbf{0} \diamond \rho\} \vdash \mathbf{0} \delta$. Since $\diamond \Sigma \subseteq \Phi, \mathbf{0} \Phi \vdash \mathbf{0} \delta$. Since $\Phi$ is $\mathbf{0 I K}$-closed, $\mathbf{0} \delta \in \Gamma$. So 0 satisfies (ii). Since $\diamond \Sigma \subseteq \Phi, 0$ satisfies (iii). 0 vacuously satisfies (iv).

Induction step. Given $j$, assume the obvious IH. Fix $n$ so that $2 n \leq j \leq 2 n+1$. Assume that $j=2 n$. So $2 n+1 \in q$. If $\Gamma \cup 1 \Psi_{2 n} \nvdash 1 \zeta_{n}$, the IH insures that $2 n+1$ satisfies (ii)-(iii), it vacuously satisifes (iv), and $2 n+2$ trivially satisifes (i)(iv). Assume that $\Gamma \cup \mathbf{1} \Psi_{2 n} \vdash \mathbf{1} \zeta_{n}$. Given $\delta \in F m l$, assume that $\Gamma \cup \mathbf{1} \Psi_{2 n+1} \vdash \mathbf{0} \delta$. Cutting $1 \zeta_{n}, \Gamma \cup \mathbf{1} \Psi_{2 n} \vdash \mathbf{0} \delta$; by the IH $2 n$ satisifes (ii); so $\mathbf{0} \delta \in \Gamma \cup \mathbf{1} \Psi_{2 n}$; so $\mathbf{0} \delta \in \Gamma \cup \mathbf{1} \Psi_{2 n+1}$. So $2 n+1$ satisfies (ii). Fix $\rho \in \Sigma$ so that $\mathbf{0} \Phi \cup\{\mathbf{1} \rho\} \cup \mathbf{1} \Psi_{2 n} \vdash \mathbf{1} \zeta_{n}$. Let $\psi$ be $\bigwedge \Psi_{2 n}$. Using admissible rules,

$$
\mathbf{0} \Phi \cup\{\mathbf{1} \top\} \vdash \mathbf{1}\left((\rho \& \psi) \supset\left(\rho \& \zeta_{n} \& \psi\right)\right) .
$$

Using $\square I$, with empty barrier, $\mathbf{0} \Phi \vdash \mathbf{0} \square\left((\rho \& \psi) \supset\left(\rho \& \zeta_{n} \& \psi\right)\right)$. Using 4.2.(3),

$$
\mathbf{0} \Phi \cup\{\mathbf{0} \diamond(\rho \& \psi)\} \vdash \mathbf{0} \diamond\left(\rho \& \zeta_{n} \& \psi\right)
$$


By the IH, $2 n$ satisfies (iii); so $\diamond(\rho \& \psi) \in \Phi$; so $\mathbf{0} \Phi \vdash \mathbf{0} \diamond\left(\rho \& \zeta_{n} \& \psi\right)$; so $\diamond\left(\rho \& \zeta_{n} \& \psi\right) \in \Phi$. Since $\bigwedge \Psi_{2 n+1}$ is $\left(\zeta_{n} \& \psi\right), 2 n+1$ satisfies (iii). If $\zeta_{n}$ is not a disjunction, $2 n+2$ trivially satisfies (i)-(iv). Assume that $\zeta_{n}$ is $\left(\varphi_{0} \vee \varphi_{1}\right)$. Subclaim: for some $i \in 2$, $i$ satisfies $\left(*_{2 n+2}\right)$. Assume otherwise. Let $\psi^{\prime}$ be $\left(\zeta_{n} \& \psi\right)$ ). For both $i \in 2$ fix $\sigma_{i} \in \Sigma$ so that $\diamond\left(\varphi_{i} \& \sigma_{i} \& \psi^{\prime}\right) \notin \Phi$. Let $\sigma$ be $\left(\sigma_{0} \& \sigma_{1}\right)$; so $\sigma \in \Sigma$. We have shown that $\diamond\left(\sigma \& \psi^{\prime}\right) \in \Phi$. Using Necessitation and 4.2.(3),

$$
\mathbf{0} \diamond\left(\sigma \& \psi^{\prime}\right) \vdash \mathbf{0} \diamond\left(\left(\varphi_{0} \& \sigma \& \psi^{\prime}\right) \vee\left(\varphi_{1} \& \sigma \& \psi^{\prime}\right)\right) .
$$

Using 4.2.(5),

$$
\mathbf{0} \diamond\left(\left(\varphi_{0} \& \sigma \& \psi^{\prime}\right) \vee\left(\varphi_{1} \& \sigma \& \psi^{\prime}\right)\right) \vdash \mathbf{0}\left(\diamond\left(\varphi_{0} \& \sigma \& \psi^{\prime}\right) \vee \diamond\left(\varphi_{1} \& \sigma \& \psi^{\prime}\right)\right)
$$

Since $\Phi$ is $\mathbf{0}$-closed, $\diamond\left(\varphi_{0} \& \sigma \& \psi^{\prime}\right) \vee \diamond\left(\varphi_{1} \& \sigma \& \psi^{\prime}\right) \in \Phi$; since $\Phi$ is $\vee$-complete, for some $i \in 2, \diamond\left(\varphi_{i} \& \sigma \& \psi^{\prime}\right) \in \Phi$. Fix such an $i$. Check that $\mathbf{0} \diamond\left(\varphi_{i} \& \sigma \& \psi^{\prime}\right) \vdash$ $\mathbf{0} \diamond\left(\varphi_{i} \& \sigma_{i} \& \psi^{\prime}\right)$. Since $\Phi$ is $\mathbf{0}$-closed, $\diamond\left(\varphi_{i} \& \sigma_{i} \& \psi^{\prime}\right) \in \Phi$, a contradiction. The subclaim follows. Fix the $i \in 2$ for which $\Psi_{2 n+2}=\Psi_{2 n+1} \cup\left\{\varphi_{i}\right\}$. Given $\theta \in F m l$, assume that $\Gamma \cup \mathbf{1} \Psi_{2 n+1} \cup\left\{\mathbf{1} \varphi_{i}\right\} \vdash \mathbf{0} \theta$. So $\Gamma \cup\left\{\mathbf{1}\left(\varphi_{i} \& \psi^{\prime}\right)\right\} \vdash \mathbf{0} \theta$. Fix $\rho_{1}, \ldots, \rho_{m} \in \Sigma$ so that

$$
\mathbf{0} \Phi \cup\left\{\mathbf{1} \rho_{i \in(m)}\right\} \cup\left\{\mathbf{1}\left(\varphi_{i} \& \psi^{\prime}\right)\right\} \vdash \mathbf{0} \theta .
$$

Let $\rho$ be $\bigwedge_{i \in(m)} \rho_{i}$. Using $1 \& E$ and $\square E, \mathbf{0} \Phi \cup\left\{\mathbf{1}\left(\varphi_{i} \& \rho \& \psi^{\prime}\right)\right\} \vdash \mathbf{0} \theta$, witnessed by a deduction in which for some $v \in \operatorname{Var}\{\}$ is a barrier with exception for $\nu: \mathbf{1}\left(\varphi_{i} \& \rho \& \psi^{\prime}\right)$. Using $\diamond E, \Gamma \cup\left\{\mathbf{0} \diamond\left(\varphi_{i} \& \rho \& \psi^{\prime}\right)\right\} \vdash \mathbf{0} \theta$. Since $i$ satisfies $\left({ }^{*} 2 n+2\right)$, $\diamond\left(\varphi_{i} \& \rho \& \psi^{\prime}\right) \in \Phi$; so $\Gamma \vdash \mathbf{0} \theta$; since 0 satisfies (iii), $\mathbf{0} \theta \in \Gamma \cup \mathbf{1} \Psi_{2 n+2}$. So $2 n+2$ satisfies (ii). Since $i$ satisfies $(* 2 n+2), 2 n+2$ satisfies (iii) and (iv).

Claim 1 follows by induction. Thus $q=\omega$. Let $\Psi=\bigcup \Psi_{j \in \omega}$. Clearly $\Gamma \subseteq$ $\mathbf{0} \Phi \cup \mathbf{1} \Psi$; so $\Sigma \subseteq \Psi$.

Claim 2: $\mathbf{0} \Phi \cup \mathbf{1} \Psi$ avoids $\mathbf{0} \perp$. Given $\delta \in F m l$, assume that $\mathbf{0} \Phi \cup \mathbf{1} \Psi \vdash \mathbf{0} \delta$. Since $\vdash$ is finitary, we may fix $j \in \omega$ so that $\Gamma \cup \mathbf{1} \Psi_{j} \vdash \mathbf{0} \delta$. By Claim 1.(ii), $\mathbf{0} \delta \in \Gamma \cup \mathbf{1} \Psi_{j}$. Thus $\mathbf{0} \Phi \cup \mathbf{1} \Psi$ is $\mathbf{0}$-closed. Assume that $\mathbf{0} \Phi \cup \mathbf{1} \Psi \vdash \mathbf{1} \delta$. By the infinite visitation condition we may fix $n$ so that $\zeta_{n}$ is $\delta$ and $\Gamma \cup 1 \Psi_{2 n} \vdash \mathbf{1} \delta$. So $\delta \in \Psi_{2 n+1}$; so $\mathbf{1} \delta \in \mathbf{0} \Phi \cup \mathbf{1} \Psi$. So $\mathbf{0} \Phi \cup \mathbf{1} \Psi$ is $\mathbf{1}$-closed. If $\mathbf{0} \perp \in \mathbf{0} \Phi \cup \mathbf{1} \Psi, \perp \in \Phi$, contrary to $\Phi \in W$. Claim 2 follows.

Since $\bigwedge\{\}$ is $T$ and $\Sigma$ is closed under conjunction, $T \in \Sigma$; so $\diamond \top \in \Psi$; so $\Psi \neq\{\}$. By the $\mathbf{0} \perp$-Avoidance Lemma, $\Phi R \Psi$.

\subsection{The Right Lemma for $\vdash$}

If $\Sigma_{0} \sqsubseteq \Sigma_{0}^{\prime}$ and $\Sigma_{0} R \Sigma_{1}$ then for some $\Sigma_{1}^{\prime} \in W \Sigma_{0}^{\prime} R \Sigma_{1}^{\prime}$ and $\Sigma_{1} \sqsubseteq \Sigma_{1}^{\prime}$.

Proof. Assume the if-clause. Since $\Sigma_{1} \in W, \mathbf{0} \Sigma_{1}$ is $-\mathbf{0}$-closed ${ }_{\vdash}$, and so $\Sigma_{1}$ is closed under conjunction. Also $\diamond \Sigma_{1} \subseteq \Sigma_{0}^{\prime}$. Take $\Sigma_{0}^{\prime}$ and $\Sigma_{1}$ to be $\Phi$ and $\Sigma$ in the statement of the Diamond Lemma. So the Diamond lemma yields a $\Sigma_{1}^{\prime}$ (called $\Psi$ in the statement of that lemma) which is as required.

\subsection{Corollary}

$F_{\vdash}$ is a IK-frame. 
Proof: the Left Lemma yields left completeness, and the Right Lemma yields right completeness of $F_{\vdash}$.

\subsection{Definitions}

For $\Sigma \in W_{\vdash}$ and $\gamma \in S$, let

$$
\mathcal{V}_{\vdash}(\Sigma, \gamma)=\left\{\begin{array}{l}
1 \text { if } \gamma \in \Sigma, \\
0 \text { otherwise }
\end{array}\right.
$$

$\mathcal{M}_{\vdash}=\left\langle F_{\vdash}, \mathcal{V}_{\vdash}\right\rangle$ is the canonical model for $\vdash$. By 7.16, it is an IK-model. Where confusion is unlikely, I will omit the subscript.

Let the canonical IK-model based on $S$ be $\mathcal{M}_{\vdash_{I K}}$.

\subsection{The Canonical Model Theorem for $\vdash$}

For any formula $\varphi$ based on $S$, for any $\Sigma \in W(*) \mathcal{M}_{\vdash}, \Sigma \models \varphi$ iff $\varphi \in \Sigma$.

Proof by induction on the construction of formulas. The base step is trivial. So are some of the cases under the induction step. We'll consider the less-trivial cases under the induction step.

Assume that $\varphi$ is $\left(\varphi_{0} \supset \varphi_{1}\right)$. Consider any $\Phi \in W$. Assume that $\mathcal{M}, \Phi \models \varphi$. Let $\Psi=\Phi \cup\left\{\varphi_{0}\right\}$. Claim: $\mathbf{0} \Psi \vdash \mathbf{0} \varphi_{1}$. Assume not. By Lindenbaum's Lemma, we may fix a $\Phi^{\prime} \in W$ so that $\Psi \subseteq \Phi^{\prime}$ and $\varphi_{1} \notin \Phi^{\prime}$. Since $\varphi_{0} \in \Phi^{\prime}$, by the IH $\mathcal{M}, \Phi^{\prime} \models \varphi_{0}$. Since $\Phi \subseteq \Phi^{\prime}$, by the Persistence Lemma $\mathcal{M}, \Phi^{\prime} \models \varphi_{1}$. By the IH, $\varphi_{1} \in \Phi^{\prime}$, a contradiction. The claim follows. Since $\mathbf{0} \Phi \cup\left\{\mathbf{0} \varphi_{0}\right\} \vdash \mathbf{0} \varphi_{1}, \mathbf{0} \Phi \vdash \mathbf{0} \varphi$; since $\Phi$ is 0-closed, o $\mathbf{0} \varphi \in \mathbf{0} \Phi$; so $\varphi \in \Phi$. Now assume that $\varphi \in \Phi$. Consider any $\Phi^{\prime}$ so that $\Phi \sqsubseteq \Phi^{\prime}$. Clearly $\varphi \in \Phi^{\prime}$. Assume that $\mathcal{M}, \Phi^{\prime}=\varphi_{0}$. By the $\mathrm{IH}, \varphi_{0} \in \Phi^{\prime}$. Since $\left\{\boldsymbol{0} \varphi_{0}, \boldsymbol{0} \varphi\right\} \vdash \mathbf{0} \varphi_{1}$ and $\mathbf{0} \Phi^{\prime}$ is $\mathbf{0}$-closed ${ }_{\vdash}, \mathbf{0} \varphi_{1} \in \mathbf{0} \Phi^{\prime}$; so $\varphi_{1} \in \Phi^{\prime}$. By the IH, $\mathcal{M}, \Phi^{\prime} \models \varphi_{1}$. Thus (*).

Assume that $\varphi$ is $\nabla \varphi_{0}$. Consider any $\Phi \in W$. Assume that $\mathcal{M}, \Phi \models \varphi$. Fix a $\Phi_{1} \in W$ so that $\Phi R \Phi_{1}$ and $\mathcal{M}, \Phi_{1}=\varphi_{0}$. By the IH, $\varphi_{0} \in \Phi_{1}$. So $\mathbf{1} \varphi_{0} \in \mathbf{0} \Phi \cup \mathbf{1} \Phi_{1}$. Using $\diamond I, \mathbf{0} \Phi \cup \mathbf{1} \Phi_{1} \vdash \mathbf{0} \diamond \varphi_{0}$. By the $\mathbf{0} \perp$-Avoidance Lemma, $\mathbf{0} \Phi \cup \mathbf{1} \Phi_{1}$ is $\mathbf{0}$-closed - $_{\vdash}$; so $\mathbf{0} \varphi \in \mathbf{0} \Phi \cup \mathbf{1} \Phi_{1}$; so $\varphi \in \Phi$. Now assume that $\varphi \in \Phi$. Let $\Sigma=$ the closure of $\left\{\varphi_{0}\right\}$ under conjunction. For every $n \in \omega$, let $\varphi^{\prime}$ be the $n$-fold conjunction of $\varphi_{0}$; check that $\nabla \varphi^{\prime} \in \Phi$. By the Diamond Lemma we may fix a $\Phi_{1}$ such that $\Sigma \subseteq \Phi_{1}$ and $\Phi R \Phi_{1}$. Since $\varphi_{0} \in \Phi_{1}$, by the IH, $\mathcal{M}, \Phi_{1} \models \varphi_{0}$. So $\mathcal{M}, \Phi \models \varphi$. Thus (*).

Assume that $\varphi$ is $\square \varphi_{0}$. Consider any $\Phi \in W$. Assume that $\mathcal{M}, \Phi \models \varphi$. Case 1: $\mathbf{0} \Phi \cup\{\mathbf{1} \top\} \vdash \mathbf{1} \varphi_{0}$. Using $\square I$, with empty barrier, $\mathbf{0} \Phi \vdash \mathbf{0} \square \varphi_{0}$. So $\varphi \in \Phi$. Case 2: otherwise. Claim: $\mathbf{0} \Phi \vdash \mathbf{1} \varphi_{0}$. Assume not. By the Avoidance Theorem there is a $\Phi_{0} \in W$ and a $\Phi_{1} \subseteq F m l$ so that $\Phi \sqsubseteq \Phi_{0}$ and $\mathbf{0} \Phi_{0} \cup 1 \Phi_{1}$ avoids $1 \varphi_{0}$; by case assumption and the furthermore-clause of that theorem, $\Phi_{0} R \Phi_{1}$. If $\varphi_{0} \in \Phi_{1}$ then $\mathbf{1} \varphi_{0} \in \mathbf{0} \Phi_{0} \cup \mathbf{1} \Phi_{1}$, contrary to avoidance; so $\varphi_{0} \notin \Phi_{1}$. By the $\mathrm{IH}, \mathcal{M}, \Phi_{1} \not \models \varphi_{0}$. Since $\Phi R^{+} \Phi_{1}$, this contradicts $\mathcal{M}, \Phi \models \varphi$. The claim follows. Using $\square I, \mathbf{0} \Phi \vdash \mathbf{0} \square \varphi_{0}$. Since $\Phi \in W, \mathbf{0} \Phi$ is $\mathbf{0}$-closed $\vdash$; so $\varphi \in \Phi$. Now assume that $\varphi \in \Phi$. Consider any $\Phi_{0}$ and $\Phi_{1}$ so that $\Phi \sqsubseteq \Phi_{0} R \Phi_{1}$. Using $\square E,\{\mathbf{0} \varphi, \mathbf{1} \top\} \vdash \mathbf{1} \varphi_{0}$, Since $\top \in \Phi_{1}$, 
$\mathbf{0} \Phi_{0} \cup \mathbf{1} \Phi_{1} \vdash \mathbf{1} \varphi_{0}$. By the $\mathbf{0} \perp$-Avoidance Lemma, $\mathbf{0} \Phi_{0} \cup \mathbf{1} \Phi_{1}$ is $\mathbf{0}$-closed $\vdash$; so $\varphi_{0} \in$ $\Phi_{1}$. By the IH, $\mathcal{M}, \Phi_{1} \models \varphi_{0}$. Thus $\mathcal{M}, \Phi \models \varphi$. Thus (*).

The theorem follows by induction.

\subsection{Completeness Theorem for $\vdash_{I K}$ (with respect to IK-models) ${ }^{30}$}

For every $\Gamma \subseteq M F m l$ and $\chi \in M F m l$, if $\langle\Gamma, \chi\rangle$ is IK-valid then $\Gamma \vdash_{I K} \chi$.

Proof. Let $\mathcal{M}=$ the canonical IK-model. Given $\Gamma$ and $\chi$, assume the if-clause. Assume that $\Gamma \nvdash_{I K} \chi$. Applying the Avoidance Theorem to $\vdash_{I K}$, fix $\Sigma_{0} \in W$ and $\Sigma_{1} \subseteq F m l$ so that $\mathbf{0} \Sigma_{0} \cup \mathbf{1} \Sigma_{1}$ avoids $\chi$ and $\Gamma \subseteq \mathbf{0} \Sigma_{0} \cup \mathbf{1} \Sigma_{1}$. By the Canonical Model Lemma $\mathcal{M}, \Sigma_{0}=\Sigma_{0}$. Case 1: $\Sigma_{1}=\{\}$. So $\Gamma \subseteq \mathbf{0 F m l}$. If $\diamond \top \in \Sigma_{0}$, $\top \in \Sigma_{1}$ (using $\diamond E^{+}$) for a contradiction; so $\Sigma_{0}$ is a dead-end in the canonical frame for $\vdash_{I K}$. Thus $\mathcal{M}, \Sigma_{0} \Vdash \mathbf{0} \Sigma_{0}$; so $\mathcal{M}, \Sigma_{0} \Vdash \Gamma$; so $\chi \in \mathbf{0} F m l$ and $\mathcal{M}, \Sigma_{0} \Vdash \chi$. Let $\chi$ be $\mathbf{0} \theta$. So $\mathcal{M}, \Sigma_{0}=\theta$. By the Canonical Model Lemma, $\theta \in \Sigma_{0}$; so $\chi \in \mathbf{0} \Sigma_{0}$, a contradiction. Case 2: otherwise. By the Avoidance Theorem (the furthermoreclause), $\Sigma_{0} R \Sigma_{1}$. Since $\Sigma_{1} \in W$, the Canonical Model Lemma entails that $\mathcal{M}, \Sigma_{1} \models$ $\Sigma_{1}$. Thus $\mathcal{M}, \Sigma_{0}, \Sigma_{1} \Vdash \mathbf{0} \Sigma_{0} \cup \mathbf{1} \Sigma_{1}$; so $\mathcal{M}, \Sigma_{0}, \Sigma_{1} \Vdash \Gamma$; so $\mathcal{M}, \Sigma_{0}, \Sigma_{1} \Vdash \chi$. Let $\chi$ be $\boldsymbol{m} \theta$. Assume that $\boldsymbol{m}$ is $\mathbf{0}$. So $\mathcal{M}, \Sigma_{0} \models \theta$; by the Canonical Model Lemma $\theta \in \Sigma_{0}$; so $\chi \in \mathbf{0} \Sigma_{0}$, a contradiction. Assume that $\boldsymbol{m}$ is $\mathbf{1}$. So $\mathcal{M}, \Sigma_{1}=\theta$; by the Canonical Model Lemma $\theta \in \Sigma_{1}$; so $\chi \in \mathbf{1} \Sigma_{1}$, a contradiction. Since both cases yield contradictions, $\Gamma \vdash_{I K} \chi$.

\subsection{Completeness Theorem for $\vdash_{I K}^{-}$(with respect to IK-models)}

For any $\Gamma \subseteq M F m l$ and $\chi \in M F m l$, if $\langle\Gamma, \chi\rangle$ is IK-valid ${ }^{-}$then $\Gamma \vdash_{I K}^{-} \chi$.

Proof. Given $\Gamma$ and $\chi$ as described, assume the if-clause. Case 1: $\Gamma \cup\{\chi\} \subseteq \mathbf{0} F \mathrm{ml}$. So $\langle\Gamma, \chi\rangle$ is IK-valid. By $7.18 \Gamma \vdash_{I K} \chi$. By 5.14, $\Gamma \vdash_{I K}^{-} \chi$. Case 2: $\Gamma \subseteq \mathbf{0} F m l$ and $\chi \in \mathbf{1 F m l}$. By 2.7.2.(4) $\langle\Gamma, \mathbf{0} \perp\rangle$ is $\mathrm{IK}_{\text {-valid }}{ }^{-}$, and so is also IK-valid. By 7.18 $\Gamma \vdash_{I K} \mathbf{0} \perp$; by $5.14 \Gamma \vdash_{I K}^{-} \mathbf{0} \perp$. With one use of $\mathbf{0} \perp E$ we can witness $\Gamma \vdash{ }_{I K}^{-} \chi$. Case 3: $\Gamma \nsubseteq \mathbf{0 F m l}$. Assume that $\Gamma \nvdash_{I K}^{-} \chi$. Claim: $\Gamma \nvdash_{I K} \chi$. Assume that $\Gamma \vdash_{I K} \chi$. Replace any use of $\diamond E^{+}$in a witnessing deduction by assumption of $1 \top$ to obtain a witness that $\Gamma \cup\{\mathbf{1} \top\} \vdash_{I K}^{-} \chi$. By the case assumption, $\Gamma \vdash_{I K}^{-} \mathbf{1} \top$. So $\Gamma \vdash_{I K}^{-} \chi$, a contradiction that yields the claim. By 7.18, $\langle\Gamma, \chi\rangle$ is not IK-valid. By 2.7.1.(1,2), $\langle\Gamma, \chi\rangle$ is not IK-valid ${ }^{-}$, a contradiction. So $\Gamma \vdash_{I K}^{-} \chi$.

\section{Classical K}

We can form a one-step version of Classical K by supplementing IK with any of the usual classicalizing rules or axioms, for example, rule $\mathbf{0}$-Excluded Middle.

\footnotetext{
${ }^{30}$ This is inference- (sometimes called strong-) completeness, as opposed to formula- (sometimes called weak) completeness.
} 


\subsection{Definition}

Define $\Rightarrow_{C K}\left[\Rightarrow_{C K}^{-}\right]$by adding the following rule to those defining $\Rightarrow_{I K}\left[\Rightarrow_{I K}^{-}\right]$.

0-Excluded Middle If $C_{0}, v_{0}: \mathbf{0} \varphi \Rightarrow_{C K} \quad \mathcal{D}_{0}: \chi, C_{1}, v_{1}: \mathbf{0} \neg \varphi \Rightarrow_{C K} \quad \mathcal{D}_{1}: \chi$, and $\left\{C_{0}, C_{1}\right\}$ is coherent, then $C_{0} \cup C_{1} \Rightarrow_{C K} \mathcal{D}: \chi$, for $\mathcal{D}$ as pictured.

$$
\begin{array}{cc}
{\left[\nu_{0}: \mathbf{0} \varphi\right]} & {\left[v_{1}: \mathbf{0} \neg \varphi\right]} \\
\mathcal{D}_{0} & \mathcal{D}_{1} \\
\chi & \chi_{\mathbf{0} E M} \\
\hline \chi^{v_{0}, v_{1}} &
\end{array}
$$

Let $\operatorname{dpd}(\mathcal{D})=\left(\operatorname{dpd}\left(\mathcal{D}_{0}\right)-\left\{s \mid \mathcal{D}_{0}(s)=v_{0}: \mathbf{0} \varphi\right\}\right) \cup\left(d p d\left(\mathcal{D}_{1}\right)-\left\{s \mid \mathcal{D}_{1}(s)=\right.\right.$ $\left.\left.v_{0}: \mathbf{0} \neg \varphi\right\}\right)$.

Define $\vdash_{C K}\left[\vdash_{C K}^{-}\right]$from $\Rightarrow_{C K}\left[\Rightarrow_{C K}^{-}\right]$in the obvious way.

\subsection{Observation}

Consider a set $\Gamma$ of formulas and a formula $\varphi$ containing no occurrences of $\square$ or $\diamond$, and an classical ND deduction $\mathcal{D}$ of $\varphi$ from $\Gamma$ using the familiar "no step" introduction and elimination rules, and perhaps the rule of excluded middle. Form $\Gamma^{\prime}, \varphi^{\prime}$ and $\mathcal{D}^{\prime}$ from $\Gamma, \varphi$ and $\mathcal{D}$ as in 4.6. Form $\mathcal{D}^{\prime \prime}$ from $\mathcal{D}^{\prime}$ by prefixing the formula-label for each string in $\operatorname{dom}\left(\mathcal{D}^{\prime}\right)$ by $\mathbf{0}$. Then $\mathcal{D}^{\prime \prime}$ witnesses that $0 \Gamma^{\prime} \vdash_{C K} \mathbf{0} \varphi^{\prime}$.

Thus $\vdash_{C K}\left|\mathbf{0} F m l=\vdash_{C K}^{-}\right| \mathbf{0} F m l=$ the result of prefixing ' $\mathbf{0}$ ' to all formulas in the familiar "no step" consequence relation $\vdash_{K}$.

\subsection{Lemma}

Next, a derived rule.

1-Excluded Middle If $C_{0}, v_{0}: \mathbf{1} \varphi \Rightarrow_{C K} \mathcal{D}_{0}: \mathbf{1} \theta, C_{1}, v_{1}: \mathbf{1} \neg \varphi \Rightarrow_{C K} \mathcal{D}_{1}: \mathbf{1} \theta,\left\{C_{0}, C_{1}\right\}$ is coherent and $\mu \notin \operatorname{dom}\left(C_{0} \cup C_{1}\right\}$, then for some CK-deduction $\mathcal{D}$ and $\mu \in \operatorname{Var}$, $C_{0} \cup C_{1} \cup\{\mu: \mathbf{1} \top\} \Rightarrow_{C K} \mathcal{D}: \mathbf{1} \theta$.

We construct such a $\mathcal{D}$ as follows. Let $\sigma_{0}$ be $((\neg \varphi \supset \theta) \supset \theta)$, and let $\sigma_{1}$ be $\left((\varphi \supset \theta) \supset \sigma_{0}\right)$. So $\sigma_{1}$ is a classical tautology. By 8.2 we may fix a CK-deduction $\mathcal{D}^{\prime}$ such that \{\}$\Rightarrow_{C K} \mathcal{D}^{\prime}: 0 \sigma_{1}$. Fix $\mu \in \operatorname{Var}-\operatorname{dom}\left(C_{0} \cup C_{1}\right)$. Let $\mathcal{D}^{\prime \prime}$ be the following.

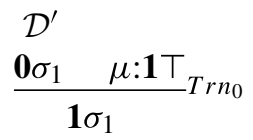

Let $\mathcal{D}_{0}^{\prime}$ and $\mathcal{D}_{1}^{\prime}$ be as follows, respectively.

\begin{tabular}{ll}
{$\left[\nu_{0}: \mathbf{1} \varphi\right]$} & {$\left[\nu_{1}: \mathbf{1} \neg \varphi\right]$} \\
$\mathcal{D}_{0}$ & $\mathcal{D}_{1}$ \\
$\mathbf{1} \theta$ & $\mu: \mathbf{1} \top$ \\
\hline $\mathbf{1}(\varphi \supset \theta)^{\nu_{0}}$ & $\frac{1 \theta}{\mathbf{1}(\neg \varphi \supset \theta)^{\nu_{1}}} \mathbf{1} \supset I$
\end{tabular}


Let $\mathcal{D}$ be the following.

$$
\begin{aligned}
& \mu: 1 \top \\
& \mathcal{D}^{\prime} \quad \mathcal{D}_{0} \\
& \frac{1 \sigma_{1} \mathbf{1}(\varphi \supset \theta)}{\frac{1 \sigma_{0}}{1} E_{1} \begin{array}{c}
\mathcal{D}_{1} \\
\mathbf{1}(\neg \varphi \supset \theta)
\end{array}} \mathbf{1} \supset E
\end{aligned}
$$

\subsection{Observations}

Next, two important schematic deductions.

(1) $\mathbf{0} \neg \diamond \neg \varphi \vdash_{C K} \mathbf{0} \square \varphi$, as witnessed by the following.

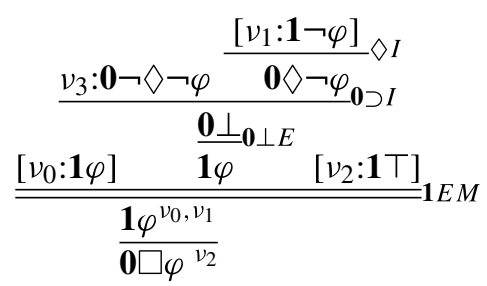

(2) $\mathbf{0} \neg \square \varphi \vdash_{C K} \mathbf{0} \diamond \neg \varphi$, as witnessed by the following, taking $\mathcal{D}^{\prime}$ to witness the previous observation.

$$
\begin{aligned}
& {\left[\nu_{3}: \mathbf{0} \neg \diamond \neg \varphi\right]} \\
& \mathcal{D}^{\prime}
\end{aligned}
$$

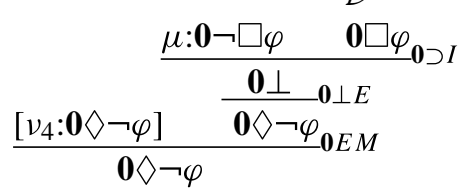

Since $\mathbf{0} \diamond \neg \varphi \vdash_{I K} \mathbf{0} \neg \square \varphi$ and $\vdash_{I K} \subseteq \vdash_{C K}, \neg \square$ and $\diamond \neg$ are equivalent under $\vdash_{C K}$. Since we also have $\mathbf{0} \neg \neg \varphi \vdash_{C K} \mathbf{0} \varphi$ and $\mathbf{0} \varphi \vdash_{C K} \mathbf{0} \neg \neg \varphi$, we have the familiar classical interdefinability of $\square$ and $\diamond .{ }^{31}$

\subsection{Definition}

A CK-frame $\langle W, R$, $\sqsubseteq\rangle$ is an IK-frame such that $\sqsubseteq=i d \mid W$. A CK-model is an IKmodel with a CK-frame. An inference $\langle\Gamma, \chi\rangle$ is $\mathrm{CK}$-valid iff it is $\mathcal{M}$-valid for every CK-model $\mathcal{M}$.

\subsection{Observation}

For any CK-model $\mathcal{M}, u \in W^{\mathcal{M}}$ and $\varphi_{i \in 2} \in F m l, \mathcal{M}, u \models\left(\varphi_{0} \supset \varphi_{1}\right)$ iff either $\mathcal{M}, u \not \models \varphi_{0}$ or $\mathcal{M}, u \models \varphi_{1}$.

\footnotetext{
${ }^{31}$ So taking $\square$ to be defined as $\neg \diamond \neg$ we could drop $\square I$ and $\square E$ in the definition of $\Rightarrow_{C K}$ without changing $\vdash_{C K}$; and taking $\diamond$ to be defined we could drop $\diamond I, \diamond E_{0}$ and $\diamond E_{1}$ with similar lack of effect.
} 


\subsection{Theorem}

$\vdash_{C K}$ is sound with respect to $\mathcal{M}$-validity for $\mathcal{M}$ a CK-model, i.e. for any $\Gamma \subseteq M F m l$ and $\chi \in M F m l$, if $\Gamma \vdash_{C K} \chi$ then $\langle\Gamma, \chi\rangle$ is CK-valid. Furthermore, $\vdash_{C K}^{-}$is sound with respect to $\mathcal{M}$-validity ${ }^{-}$.

Proof: a straightforward induction on the stages of the definition of $\Rightarrow_{C K}$ (i.e. on the height of classical deductions), using 8.6.

\subsection{Observation}

$\vdash_{C K}^{-} \subsetneq \vdash_{C K}$. The proof of 6.3 transfers.

\subsection{Definitions}

Consider a $\Gamma \subseteq M F m l . \Gamma$ is maximally CK-consistent iff $\Gamma \nvdash_{C K} \mathbf{0} \perp$, and for any $\Gamma^{\prime}$ such that $\Gamma \subseteq \Gamma^{\prime} \subseteq M F m l$, if $\Gamma^{\prime} \nvdash_{C K} \mathbf{0} \perp$ then $\Gamma=\Gamma^{\prime}$.

\subsection{Lemma}

For every $\Sigma, \Sigma \in W_{\vdash_{C K}}$ iff $\Sigma \in W_{\vdash_{C K}}$ and $\Sigma$ is maximally CK-consistent.

Proof. Given $\Sigma \in W_{\vdash_{C K}}$, this the key point: if $\Sigma$ is $\mathbf{0}$-closed $\vdash_{C K}$ then for any $\varphi \in F m l,(\varphi \vee \neg \varphi) \in \Sigma$, and thus (since $\Sigma$ is $\vee$-complete) either $\varphi \in \Sigma$ or $\neg \varphi \in \Sigma$. With that, the lemma follows by well-known arguments.

\subsection{Observation}

$F_{\vdash_{C K}}$ is a CK-frame. Proof: use the previous lemma. So $\mathcal{M}_{\vdash_{C K}}$ is a CK-model; call it the canonical CK-model based on vocabulary set $S$.

The obvious Completeness Theorem for CK follow by slight variations of the arguments used for 7.18 .

\section{References}

1. Fine, K. (1995). The Logic of Essence. Journal of Philosophical Logic, 24(3), 241-273.

2. Girard, J.-Y. (1987). Proof theory and logical complexity. Napoli: Bibliopolis.

3. Hodes, H.T. (2004). On the Sense and Reference of a Logical Constant. The Philosophical Quarterly, 54(214), 134-165.

4. Humberstone, L. (1982). Scope and Subjunctivity. Philosophia, 12(1-2), 99-126.

5. Parisi, A. (2017). Second-Order Modal logic. University of Connectituct doctoral dissertationss, 1480.

6. Peacocke, C. (1992). A study of concepts. Cambridge: MIT Press.

7. Plotkin, G., \& Stirling, C. (1986). A framework for intuitionistic modal logics. In Halpern, J.Y. (Ed.) Theoretical aspects of reasoning about knowledge (pp. 399-406). Morgan Kaufmann Publishers.

8. Turner, J. (2010). Ontological Pluralism. Journal of Philosophy, 107(1), 5-34.

9. Wehmeier, K. (2004). In the Mood. Journal of Philosophical Logic, 33(6), 607-630.

10. Wehmeier, K., \& Rückert, H. (2019). Still in the Mood. Topoi, 48(2), 361-377.

11. Wojcicki, R. (1988). Theory of logical calculi, Synthese Library. Berlin: Kluwer Academic Publishers.

Publisher's Note Springer Nature remains neutral with regard to jurisdictional claims in published maps and institutional affiliations. 